\title{
Mid-Infrared line diagnostics of active galaxies
}

\section{A spectroscopic AGN survey with ISO-SWS ${ }^{\star}$}

\author{
E. Sturm ${ }^{1}$, D. Lutz ${ }^{1}$, A. Verma ${ }^{1}$, H. Netzer ${ }^{2}$, A. Sternberg ${ }^{2}$, A. F. M. Moorwood ${ }^{3}$, E. Oliva ${ }^{4}$, and R. Genzel ${ }^{1}$ \\ 1 Max-Planck-Institut für extraterrestrische Physik, Postfach 1312, 85741 Garching, Germany \\ 2 School of Physics and Astronomy and Wise Observatory, Raymond and Beverly Sackler Faculty of Exact Sciences, \\ Tel Aviv University, Ramat Aviv, Tel Aviv 69978, Israel \\ 3 European Southern Observatory, Karl-Schwarzschild-Strasse 2, 85748 Garching, Germany \\ ${ }^{4}$ Osservatorio Astrofisico di Arcetri, Largo E. Fermi 5, 50125 Firenze, Italy, and Telescopio Nazionale Galileo \& Centro \\ Galileo Galilei, calle Alvarez de Abreu 70, 38700 Santa Cruz de La Palma, TF, Spain
}

\section{Received 1 July 2002 / Accepted 11 July 2002}

\begin{abstract}
We present medium resolution $(R \sim 1500)$ ISO-SWS $2.4-45 \mu \mathrm{m}$ spectra of a sample of 29 galaxies with active nuclei. This data set is rich in fine structure emission lines tracing the narrow line regions and (circum-)nuclear star formation regions, and it provides a coherent spectroscopic reference for future extragalactic studies in the mid-infrared. We use the data set to briefly discuss the physical conditions in the narrow line regions (density, temperature, excitation, line profiles) and to test for possible differences between AGN sub-types. Our main focus is on new tools for determining the properties of dusty galaxies and on the AGN-starburst connection. We present mid-IR line ratio diagrams which can be used to identify composite (starburst + AGN) sources and to distinguish between emission excited by active nuclei and emission from (circum-nuclear) star forming regions. For instance, line ratios of high to low excitation lines like [O IV] $25.9 \mu \mathrm{m} /[\mathrm{Ne}$ II $] 12.8 \mu \mathrm{m}$, that have been used to probe for AGNs in dusty objects, can be examined in more detail and with better statistics now. In addition, we present twodimensional diagnostic diagrams that are fully analogous to classical optical diagnostic diagrams, but better suited for objects with high extinction. Finally, we discuss correlations of mid-infrared line fluxes to the mid- and far-infrared continuum. We compare these relations to similar relations in starburst galaxies in order to examine the contribution of AGNs to the bolometric luminosities of their host galaxies. The spectra are available in electronic form from the authors.
\end{abstract}

Key words. infrared: galaxies - galaxies: active - galaxies: Seyfert - galaxies: starburst

\section{Introduction}

Mid-infrared spectra contain a large number of atomic, ionic and molecular lines along with various solid-state and dust features in grains of different sizes. They are therefore an excellent tool to study the nature of infrared bright galaxies, such as galaxies with active galactic nuclei (AGNs). For instance, mid-infrared (MIR) continua can be compared to torus models (e.g. D. Alexander et al. 1999; Siebenmorgen et al. 1997), whereas ice absorption features allow the study of the composition of the ISM and the extinction law in AGNs (Chiar et al. 2000; Spoon et al. 2002). Clavel et al. (2000) have used aromatic infrared bands (AIBs, often identified as "PAHs" Polycyclic Aromatic Hydrocarbons) as a new tool for testing unified schemes in Seyfert 1 and Seyfert 2 galaxies, and

Send offprint requests to: E. Sturm, e-mail: sturm@mpe.mpg.de

* Based on observations with ISO, an ESA project with instruments funded by ESA member states (especially the PI countries: France, Germany, The Netherlands and the UK) and with participation of ISAS and NASA.
Lutz et al. (2000a) have searched for broad line components of hidden broad line regions (BLRs). For a more complete, recent review see Genzel \& Cesarsky (2000). A good overview of the global characteristics of MIR AGN spectra (like shapes, features, relative line intensities) can be obtained from the complete 2.5 to $45 \mu \mathrm{m}$ spectra of several AGN and starburst prototypes presented in Sturm et al. (2000), in combination with the detailed and comprehensive line spectra of NGC 1068, NGC 4151, and the Circinus galaxy published in Lutz et al. (2000b), Sturm et al. (1999) and Moorwood et al. (1996).

In this paper we concentrate on narrow emission lines. The MIR fine structure emission lines cover a wide range of physical conditions, like excitation $(0-300 \mathrm{eV})$, or critical density $\left(10^{2}<n_{\mathrm{H}}<10^{7} \mathrm{~cm}^{-3}\right)$. They are significantly less affected by extinction than UV, optical or near-IR lines, and their emissivities depend only weakly on electron temperature, in contrast to their optical and UV counterparts. Low excitation ionic fine structure lines (excitation potential $\leq 50 \mathrm{eV}$ ) sample HII regions that are photoionized by massive stars 
Table 1. The sample.

\begin{tabular}{|c|c|c|c|c|c|c|c|c|c|}
\hline No. & Galaxy & Type & $z$ & $S_{12}^{\dagger}$ & $S_{25}^{\dagger}$ & $S_{60}^{\dagger}$ & $S_{100}^{\dagger}$ & $D^{\dagger \dagger}$ & $L_{\mathrm{IR}}^{\dagger \dagger \dagger}$ \\
\hline 1 & Cen A & Sy2 + SB & 0.001828 & 13.3 & 17.3 & 162 & 314 & 4 & 0.9 \\
\hline 2 & Circinus & Sy $2+$ SB & 0.001498 & 18.9 & 68.4 & 248 & 314 & 3 & 0.7 \\
\hline 3 & M 51 & Sy $2+$ SB & 0.001544 & 11.0 & 17.5 & 109 & 292 & 6 & 1.3 \\
\hline 4 & M 87 & Sy & 0.004360 & 0.2 & 0.2 & 0.4 & 1.0 & 17 & 0.1 \\
\hline 5 & Mkn 1 (=NGC 449) & Sy2 & 0.015944 & 1.9 & 0.9 & 2.5 & 2.9 & 65 & 9.5 \\
\hline 6 & Mkn 3 & Sy2 & 0.013509 & 0.7 & 2.9 & 3.8 & 3.4 & 54 & 6.1 \\
\hline 7 & Mkn 6 & Sy1.5 & 0.018813 & 0.2 & 0.7 & 1.2 & $<1.7$ & 76 & 3.6 \\
\hline 8 & Mkn 335 & Sy1.2 & 0.025785 & 0.4 & 0.3 & 0.3 & 0.5 & 104 & 4.4 \\
\hline 9 & Mkn 463 & $(2 x) \operatorname{Sy} 2$ & 0.050802 & 0.5 & 1.6 & 2.2 & 1.9 & 202 & 51.0 \\
\hline 10 & Mkn 509 & Sy 1.2 & 0.034397 & 0.3 & 0.7 & 1.4 & 1.5 & 139 & 14.0 \\
\hline 11 & Mkn 573 & Sy2 & 0.017259 & 0.3 & 0.9 & 1.2 & 1.4 & 69 & 3.5 \\
\hline 12 & Mkn 938 (=NGC 34) & Sy2 & 0.019784 & 0.4 & 2.4 & 16.6 & 17.2 & 80 & 28.0 \\
\hline 13 & Mkn 1014 & Sy1 & 0.163099 & 0.1 & 0.5 & 2.2 & 2.2 & 677 & 250.0 \\
\hline 14 & NGC 613 & Sy/Radio + SB & 0.004920 & 1.0 & 2.5 & 22 & 50 & 20 & 3.0 \\
\hline 15 & NGC 1068 & Sy2 & 0.003829 & 38.0 & 86.0 & 186 & 239 & 14 & 30.0 \\
\hline 16 & NGC 1275 & $\mathrm{Sy} 2+\mathrm{SB}$ & 0.017559 & 1.1 & 3.5 & 7.1 & 6.9 & 71 & 16.0 \\
\hline 17 & NGC 1365 & Sy $1.8+$ SB & 0.005457 & 3.3 & 1.1 & 76 & 142 & 22 & 12.0 \\
\hline 18 & NGC 3783 & Sy1 & 0.009730 & 0.8 & 2.5 & 3.3 & 4.9 & 34 & 2.5 \\
\hline 19 & NGC 4051 & Sy1.5 & 0.002418 & 0.9 & 1.6 & 7.1 & 23.9 & 10 & 0.4 \\
\hline 20 & NGC 4151 & Sy 1.5 & 0.003319 & 1.8 & 4.7 & 6.9 & 10.2 & 20 & 1.7 \\
\hline 21 & NGC 5506 & Sy1.9/NLXG & 0.006181 & 1.3 & 3.6 & 8.4 & 8.9 & 23 & 2.0 \\
\hline 22 & NGC 5643 & Sy2 & 0.003999 & 1.1 & 3.7 & 19.5 & 38.2 & 16 & 1.8 \\
\hline 23 & NGC 7469 & Sy $1.2+$ SB & 0.016398 & 1.3 & 5.8 & 25.9 & 34.9 & 65 & 29.0 \\
\hline 24 & NGC 7582 & Sy $2 / N L X G+S B$ & 0.005254 & 1.4 & 6.3 & 48 & 72.8 & 20 & 5.0 \\
\hline 25 & PKS 2048-57 & Sy 2 & 0.011348 & 1.1 & 3.9 & 4.3 & 4.2 & 45 & 6.1 \\
\hline 26 & Tol 0109-383 (=NGC 424) & Sy1.8 & 0.011661 & 1.1 & 1.7 & 1.8 & 1.8 & 47 & 3.7 \\
\hline 27 & I Zw 1 & Sy1 & 0.061086 & 0.5 & 1.2 & 2.2 & 2.6 & 248 & 71.0 \\
\hline 28 & I Zw 92 (=Mkn 477) & $\mathrm{Sy} 2+\mathrm{SB}$ & 0.037799 & 0.1 & 0.5 & 1.3 & 1.9 & 153 & 12.0 \\
\hline 29 & $3 \mathrm{C} 120$ (=II Zw 14) & Sy1 & 0.033010 & 0.3 & 0.6 & 1.3 & 2.8 & 135 & 13.5 \\
\hline
\end{tabular}

$\dagger^{\dagger}=$ IRAS (FSC) fluxes at 12, 25, 60 and $100 \mu \mathrm{m}$ in Jy.

$\dagger^{\dagger}=$ distance in $\mathrm{Mpc}$, for $H_{0}=75 \mathrm{~km} \mathrm{~s}^{-1} / \mathrm{Mpc}$ and $q_{0}=0.5$.

计 $=8-1000 \mu \mathrm{m}$ luminosity in $10^{10}\left[L_{\odot}\right], L_{\mathrm{IR}}=5.6 \times D_{\mathrm{Mpc}}^{2}\left(S_{100}+2.58 S_{60}+5.16 S_{25}+13.48 S_{12}\right)$, see Sanders \& Mirabel (1996).

(Spinoglio \& Malkan 1992; Voit 1992; Giveon et al. 2002). Some contribution from ionizing shocks is possible (e.g. Contini \& Viegas 2001). Ionic lines from species with excitation potentials up to $\approx 300 \mathrm{eV}$ sample highly ionized or "coronal" gas and require the very hard radiation fields of AGNs, or fast ionizing shocks. Electron densities can be determined from ratios like [Ne V] 14/24 $\mu \mathrm{m}$. Line ratios from different ionization stages probe the hardness of the radiation field and the ionization parameter (e.g. [Ne II], [Ne III], [Ne V], [Ne VI]), but possible contributions from nuclear starburst components have to be taken into account. Finally, hydrogen recombination lines and line ratios like [S III] $33 \mu \mathrm{m} / 18 \mu \mathrm{m}$ can be used to trace the MIR extinction.

The Infrared Space Observatory (ISO, Kessler et al. 1996) has provided access to this wealth of information. In the following we present a compilation of ISO-SWS emission line spectra and line fluxes of AGNs (Sect. 4). We use this data set to derive electron densities and temperatures in the narrow line regions (NLRs) of AGNs (Sects. 5.1 and 5.2), and we compare the line ratios of highly ionized lines to the predictions of photoionization models to constrain the spectral energy distribution (SED) of the central ionizing source and to test their consistency with AGN unification models (Sect. 5.3). Furthermore, we study line profiles and their various degrees of asymmetry and differences to the profiles of their optical counterparts (Sect. 5.4). In Sect. 6 we develop a new diagram to identify those sources in our sample where star forming regions within the SWS apertures contribute to the fluxes of low lying fine structure lines ("composite sources"). In order to establish tools for the distinction of AGNs and starbursts in distant dusty galaxies we construct mid-IR line ratio diagrams which are better suited for dusty objects than their classical optical counterparts (e.g. Baldwin et al. 1981; Veilleux \& Osterbrock 1987) in Sect. 7. Finally, we discuss correlations of mid-IR lines with far-IR fluxes in the context of the far-IR energy source and the starburst-AGN connection (Sect. 8). The rich spectrum of molecular hydrogen $\left(\mathrm{H}_{2}\right)$ in our data set is discussed in a separate paper (Rigopoulou et al. 2002). 
Table 2. Observed fine structure line fluxes and upper limits (in $10^{-20} \mathrm{~W} / \mathrm{cm}^{2}$ ).

\begin{tabular}{|c|c|c|c|c|c|c|c|c|}
\hline Galaxy & [Si IX] & [Mg VIII] & [Si IX] & [Mg IV] & {$[\mathrm{Fe} \mathrm{II}]$} & [Mg VII] & {$[\mathrm{Mg} \mathrm{V}]$} & [Ar II] \\
\hline$\lambda_{\text {rest,vac }}(\mu \mathrm{m})$ & 2.584 & 3.028 & 3.936 & 4.487 & 5.340 & 5.503 & 5.610 & 6.985 \\
\hline$E_{\text {ion }}(\mathrm{eV})^{1}$ & 303.2 & 224.9 & 303.2 & 80.1 & 7.9 & 186.5 & 109.2 & 15.8 \\
\hline Resolution ${ }^{4}$ & 100 & 120 & 150 & 130 & 250 & 230 & 230 & 190 \\
\hline Aperture $^{2}$ & $14 \times 20$ & $14 \times 20$ & $14 \times 20$ & $14 \times 20$ & $14 \times 20$ & $14 \times 20$ & $14 \times 20$ & $14 \times 20$ \\
\hline Cen A & $\ldots$ & $<0.5$ & $<0.4$ & $\ldots$ & 1.6 & 1.5 & $0.9^{3}$ & 4.9 \\
\hline Circinus & 1.7 & 6.2 & 4.9 & 1.7 & 8.3 & 8.0 & 6.7 & 27.6 \\
\hline M 51 & $\ldots$ & $<0.3$ & $\ldots$ & $\ldots$ & $\ldots$ & $\ldots$ & $<1.2$ & $\ldots$ \\
\hline M 87 & $\ldots$ & $\ldots$ & $\ldots$ & $\ldots$ & $\ldots$ & $\ldots$ & $<1.0$ & $\ldots$ \\
\hline Mkn 1 & $\ldots$ & $\ldots$ & $\ldots$ & $\ldots$ & $\ldots$ & $\ldots$ & $<2.0$ & $\ldots$ \\
\hline Mkn 3 & $\ldots$ & $\ldots$ & $\ldots$ & $\ldots$ & $\ldots$ & $\ldots$ & $\ldots$ & $\ldots$ \\
\hline Mkn 6 & $<0.6$ & $<0.5$ & $\ldots$ & $<1.5$ & $\ldots$ & $\ldots$ & $\ldots$ & $\ldots$ \\
\hline Mkn 335 & $<0.5$ & $<0.3$ & $<0.2$ & $\ldots$ & $\ldots$ & $<1.8$ & $\ldots$ & $\ldots$ \\
\hline Mkn 463 & $\ldots$ & $\ldots$ & $\ldots$ & $\ldots$ & $\ldots$ & $<0.8$ & $<0.9$ & $\ldots$ \\
\hline Mkn 509 & $\ldots$ & 0.4 & $\ldots$ & $\ldots$ & $\ldots$ & $\ldots$ & $\ldots$ & $\ldots$ \\
\hline Mkn 573 & $<0.8$ & 0.6 & $\ldots$ & $\ldots$ & $\ldots$ & $\ldots$ & $<1.0$ & $\ldots$ \\
\hline Mkn 938 & $<1.4$ & $\ldots$ & $\ldots$ & $\ldots$ & $\ldots$ & $\ldots$ & $\ldots$ & $\ldots$ \\
\hline Mkn 1014 & $\ldots$ & $<1.3$ & $\ldots$ & $\ldots$ & $\ldots$ & $\ldots$ & $<1.5$ & $\ldots$ \\
\hline NGC 613 & $\ldots$ & $\ldots$ & $\ldots$ & $\ldots$ & $\ldots$ & $\ldots$ & $\ldots$ & $\ldots$ \\
\hline NGC 1068 & 3.0 & 11.0 & 5.4 & 7.4 & 5.0 & 13.0 & 18.0 & 13.0 \\
\hline NGC 1275 & $<0.7$ & $\ldots$ & $<0.2$ & $\ldots$ & $<1.0$ & $\ldots$ & $<1.3$ & 0.6 \\
\hline NGC 1365 & $<0.5$ & $<0.3$ & $<0.3$ & $<1.5$ & $\ldots$ & $<1.8$ & $<1.5$ & 14.1 \\
\hline NGC 3783 & $\ldots$ & $\ldots$ & $\ldots$ & $\ldots$ & $\ldots$ & $\ldots$ & $<1.0$ & $\ldots$ \\
\hline NGC 4051 & $\ldots$ & $\ldots$ & $\ldots$ & $\ldots$ & $\ldots$ & $\ldots$ & $\ldots$ & $\ldots$ \\
\hline NGC 4151 & 0.2 & 0.6 & 0.4 & 0.3 & $\ldots$ & $<1.0$ & $<1.5$ & $\ldots$ \\
\hline NGC 5506 & $<1.4$ & $<0.4$ & $<0.3$ & $<1.8$ & $<2.0$ & $<2.4$ & $<1.8$ & 3.2 \\
\hline NGC 5643 & $\ldots$ & $\ldots$ & $\ldots$ & $\ldots$ & $\ldots$ & $\ldots$ & $<2.0$ & $\ldots$ \\
\hline NGC 7469 & $<0.8$ & $<0.2$ & $<0.4$ & $<1.0$ & $\ldots$ & $<2.3$ & $<2.5$ & 6.7 \\
\hline NGC 7582 & $<0.5$ & $<0.3$ & $<0.3$ & $<1.2$ & $<1.5$ & $<0.9$ & $<1.5$ & $\ldots$ \\
\hline PKS 2048-57 & $\ldots$ & 0.4 & 0.2 & $\ldots$ & $\ldots$ & $<1.4$ & $<1.4$ & $\ldots$ \\
\hline TOL 0109-383 & $\ldots$ & $<0.2$ & $<0.2$ & $\ldots$ & $\ldots$ & $<1.1$ & $\ldots$ & $\ldots$ \\
\hline I Zw 1 & $\ldots$ & $\ldots$ & $\ldots$ & $\ldots$ & $\ldots$ & $\ldots$ & $<0.6$ & $\ldots$ \\
\hline I Zw 92 & $<0.4$ & $\ldots$ & $\ldots$ & $<1.5$ & $\ldots$ & $\ldots$ & $\ldots$ & $\ldots$ \\
\hline $3 \mathrm{C} 120$ & $<0.4$ & $<0.4$ & $\ldots$ & $<0.8$ & $\ldots$ & $\ldots$ & $\ldots$ & $\ldots$ \\
\hline
\end{tabular}

${ }^{1}$ Lower ionization potential of the stage leading to the transition.

${ }^{2}$ In $\operatorname{arcsec}$.

${ }^{3}$ Uncertain detection, either because of noise $(\approx 3 \sigma)$ or offset in wavelength larger than $100 \mathrm{~km} \mathrm{~s}^{-1}$ compared to similar lines in the same object.

${ }^{4} \mathrm{In} \mathrm{km} \mathrm{s}^{-1}$.

\section{Target selection}

Most of the objects discussed here have been observed as part of a guaranteed time ISO project on bright galactic nuclei (MPEXGAL), expanded by a solicited follow-up proposal (ZZCORLIN). They are well studied, nearby Seyfert galaxies, selected only on the basis of their brightness and visibility to ISO. The sample is therefore not complete in any sense. From the ISO archive we have added 9 targets which have been observed in other programmes, but for which fluxes have not been published to date. For three of these additional sources relative line strengths have been previously published and studied by Spinoglio et al. (2000). Table 1 lists the names and classifications of all 29 galaxies of our sample. According to the classifications given in the NASA/IPAC Extragalactic
Database (NED) the sample consists of 13 Seyfert 1s (of various sub-types) and 14 Seyfert 2s. NGC 5506 and NGC 7582 are classified as NLXGs. No Seyfert type is given for M 87 and NGC 613. For 8 additional Seyfert galaxies (from the CfA sample) SWS fluxes or flux limits of 4 coronal lines can be found in Prieto \& Viegas (2000).

Many active galaxies exhibit intense star formation in the (circum-)nuclear region. In these cases some fraction of the measured fluxes of low lying fine structure lines (excitation potential $\leq 50 \mathrm{eV}$ ) will be produced by photoionization from stars rather than from the AGN. In Table 1 we have indicated (Type = "Sy + SB") such composite sources where star formation regions within the SWS apertures are important. This classification will be discussed in Sect. 6 . 
Table 2. continued.

\begin{tabular}{|c|c|c|c|c|c|c|c|c|c|c|}
\hline Galaxy & {$[\mathrm{Ne} \mathrm{VI}]$} & [Fe VII] & [Ar III]/[Mg VII] & [Fe VII] & [S IV] & {$[\mathrm{Ne} \mathrm{II]}$} & {$[\mathrm{Ne} \mathrm{V}]$} & [Ne III] & [P III] & [Fe II] \\
\hline$\lambda_{\text {rest,vac }}(\mu \mathrm{m})$ & 7.652 & 7.815 & 8.991 & 9.527 & 10.511 & 12.814 & 14.322 & 15.555 & 17.885 & 17.936 \\
\hline$E_{\text {ion }}(\mathrm{eV})^{1}$ & 126.2 & 99.1 & $27.6 / 186.5$ & 99.1 & 34.8 & 21.6 & 97.1 & 41.0 & 19.8 & 7.9 \\
\hline Resolution $^{4}$ & 165 & 160 & 150 & 140 & 115 & 190 & 160 & 150 & 125 & 125 \\
\hline Aperture $^{2}$ & $14 \times 20$ & $14 \times 20$ & $14 \times 20$ & $14 \times 20$ & $14 \times 20$ & $14 \times 27$ & $14 \times 27$ & $14 \times 27$ & $14 \times 27$ & $14 \times 27$ \\
\hline Cen A & 2.0 & $\ldots$ & 0.9 & $\ldots$ & 1.4 & 22.1 & 2.7 & 14.1 & 0.4 & 0.2 \\
\hline Circinus & 36.3 & 1.5 & 8.3 & 1.1 & 12.7 & 90.0 & 31.7 & 33.5 & $1.3^{3}$ & $<1.0$ \\
\hline M 51 & $<0.3$ & $\ldots$ & $<0.5$ & $\ldots$ & $<0.8$ & 7.0 & $<0.2$ & 2.9 & $\ldots$ & $\ldots$ \\
\hline M 87 & $\ldots$ & $\ldots$ & $<0.4$ & $\ldots$ & $\ldots$ & $\ldots$ & $\ldots$ & $\ldots$ & $\ldots$ & $\ldots$ \\
\hline Mkn 1 & $\ldots$ & $\ldots$ & $<0.9$ & $\ldots$ & $\ldots$ & $\ldots$ & $\ldots$ & $\ldots$ & $\ldots$ & $\ldots$ \\
\hline Mkn 3 & 4.5 & $\ldots$ & $\ldots$ & $\ldots$ & $\ldots$ & 4.7 & 4.6 & 12.3 & $\ldots$ & $\ldots$ \\
\hline Mkn 6 & $<0.5$ & $\ldots$ & $\ldots$ & $\ldots$ & $\ldots$ & 0.8 & $<0.6$ & $1.8^{3}$ & $\ldots$ & $\ldots$ \\
\hline Mkn 335 & $<0.3$ & $\ldots$ & $\ldots$ & $\ldots$ & $\ldots$ & $<0.7$ & $<0.5$ & $<0.8$ & $\ldots$ & $\ldots$ \\
\hline Mkn 463 & $\ldots$ & $\ldots$ & 0.3 & $\ldots$ & $\ldots$ & 1.3 & 1.4 & $\ldots$ & $\ldots$ & $\ldots$ \\
\hline Mkn 509 & 0.4 & $\ldots$ & $\ldots$ & $\ldots$ & $\ldots$ & 2.0 & $\ldots$ & $\ldots$ & $\ldots$ & $\ldots$ \\
\hline Mkn 573 & 1.2 & $\ldots$ & $\ldots$ & $\ldots$ & $\ldots$ & $<1.3$ & 1.8 & 2.4 & $\ldots$ & $\ldots$ \\
\hline Mkn 938 & $\ldots$ & $\ldots$ & $\ldots$ & $\ldots$ & $\ldots$ & 5.7 & $<0.4$ & $<1.0$ & $\ldots$ & $\ldots$ \\
\hline Mkn 1014 & $\ldots$ & $\ldots$ & $\ldots$ & $\ldots$ & $\ldots$ & $0.4^{3}$ & $<0.4$ & $\ldots$ & $\ldots$ & $\ldots$ \\
\hline NGC 613 & $<0.3$ & $\ldots$ & $\ldots$ & $\ldots$ & $\ldots$ & 4.0 & $<0.8$ & $<6.8$ & $\ldots$ & $\ldots$ \\
\hline NGC 1068 & 110.0 & 3.0 & 25.0 & 4.0 & 58.0 & 70.0 & 97.0 & 160.0 & $\ldots$ & $<10.0$ \\
\hline NGC 1275 & $<0.5$ & $\ldots$ & $<0.5$ & $\ldots$ & $<0.3$ & 2.9 & $<0.7$ & 1.0 & $0.4^{3}$ & $<0.7$ \\
\hline NGC 1365 & 2.5 & $\ldots$ & 1.1 & $\ldots$ & 2.6 & 40.9 & 2.5 & 7.7 & $\ldots$ & $\ldots$ \\
\hline NGC 3783 & $\ldots$ & $\ldots$ & $0.5^{3}$ & $\ldots$ & $\ldots$ & $\ldots$ & 2.1 & $\ldots$ & $\ldots$ & $\ldots$ \\
\hline NGC 4051 & $\ldots$ & $\ldots$ & $\ldots$ & $\ldots$ & $\ldots$ & 2.5 & $<1.0$ & 3.0 & $\ldots$ & $\ldots$ \\
\hline NGC 4151 & 7.8 & $\ldots$ & 2.2 & $\ldots$ & 11.3 & 11.8 & 5.5 & 20.7 & $\ldots$ & $\ldots$ \\
\hline NGC 5506 & 2.2 & $\ldots$ & $0.7^{3}$ & $\ldots$ & 5.4 & 5.9 & 2.6 & 5.8 & $\ldots$ & $\ldots$ \\
\hline NGC 5643 & $\ldots$ & $\ldots$ & 0.9 & $\ldots$ & $\ldots$ & $\ldots$ & $\ldots$ & $\ldots$ & $\ldots$ & $\ldots$ \\
\hline NGC 7469 & 1.1 & $\ldots$ & $0.5^{3}$ & $\ldots$ & 0.9 & 22.6 & $<1.5$ & 2.2 & $\ldots$ & $\ldots$ \\
\hline NGC 7582 & 2.3 & $\ldots$ & 1.0 & $\ldots$ & 1.8 & 14.8 & 2.2 & 6.7 & $\ldots$ & $<0.6$ \\
\hline PKS 2048-57 & 2.8 & $\ldots$ & 1.0 & $\ldots$ & $\ldots$ & $2.1^{3}$ & 3.6 & 5.9 & $\ldots$ & $\ldots$ \\
\hline TOL 0109-383 & 1.2 & $\ldots$ & $\ldots$ & $\ldots$ &.. & $<1.2$ & 0.8 & $<1.6$ & $\ldots$ & $\ldots$ \\
\hline I Zw 1 & $<0.3$ & $\ldots$ & $\ldots$ & $\ldots$ & $\ldots$ & 0.65 & 0.27 & $\ldots$ & $\ldots$ & $\ldots$ \\
\hline I Zw 92 & $<0.7$ & $\ldots$ & $\ldots$ & $\ldots$ & $\ldots$ & 2.4 & $<1.5$ & 1.6 & $\ldots$ & $\ldots$ \\
\hline $3 \mathrm{C} 120$ & 1.7 & $\ldots$ & $\ldots$ & $\ldots$ & $\ldots$ & 0.9 & 1.9 & 2.0 & $\ldots$ & $\ldots$ \\
\hline
\end{tabular}

\section{Observation and data processing}

The data have been obtained with the Short-Wavelength Spectrometer (SWS, de Graauw et al. 1996) on board the Infrared Space Observatory (ISO), using the SWS02 and SWS06 observation modes. These modes provide line spectra with a resolving power of approximately 1000-2500 in the range between 2.4 and $45 \mu \mathrm{m}$. For Circinus and NGC 1068 a few lines have been added from SWS01 measurements with reduced resolving power. The observations were centered on the nuclei. Total observation times per source ranged from $10 \mathrm{~min}$ to 7 hours. Spectral lines at different wavelengths are observed with three different aperture sizes, varying between $14^{\prime \prime} \times 20^{\prime \prime}$ and $20^{\prime \prime} \times 33^{\prime \prime}$ ( see Table 2).

We have processed the data using the SWS Interactive Analysis (IA) system (Lahuis et al. 1998; Wieprecht et al. 1998) and the ISO Spectral Analysis Package ISAP (Sturm et al. 1998) together with the associated calibration files available at the end of 2000/beginning of 2001. Dark current subtraction, scan direction matching, and flatfielding have been done interactively, and noisy detectors have been eliminated. Outliers were clipped, and the data of all 12 detectors were averaged for each line, retaining the instrumental resolution. In a few cases some wavelength ranges were affected by fringes. In these cases we have defringed their averaged spectra using the FFT or iterative sine fitting options of the defringe module within ISAP. The resulting average flux calibration uncertainty is about $20 \%$.

\section{Results}

The spectra are displayed in Figs. 17 to 23. The fine structure emission lines are generally resolved, having widths (FWHM) in the range between 200 and $600 \mathrm{~km} \mathrm{~s}^{-1}$, i.e. line widths typical of narrow line regions of Seyfert galaxies. Measured line fluxes and upper limits are summarized in Table 2 (fine 
Table 2. continued.

\begin{tabular}{|c|c|c|c|c|c|c|c|c|c|c|c|}
\hline Galaxy & [S III] & [Fe III] & {$[\mathrm{Fe} \mathrm{I}]$} & {$[\mathrm{Ne} \mathrm{V}]$} & [S I] & [O IV] & {$[\mathrm{Fe} \mathrm{II}]$} & [S III] & [Si II] & [Fe II] & [Ne III] \\
\hline$\lambda_{\text {rest,vac }}(\mu \mathrm{m})$ & 18.713 & 22.925 & 24.042 & 24.318 & 25.249 & 25.890 & 25.988 & 33.481 & 34.815 & 35.349 & 36.014 \\
\hline$E_{\text {ion }}(\mathrm{eV})^{1}$ & 23.3 & 16.2 & 0.0 & 97.1 & 0.0 & 54.9 & 7.9 & 23.3 & 8.2 & 7.9 & 41.0 \\
\hline Resolution $^{4}$ & 120 & 250 & 230 & 230 & 225 & 215 & 215 & 195 & 185 & 185 & 185 \\
\hline Aperture $^{2}$ & $14 \times 27$ & $14 \times 27$ & $14 \times 27$ & $14 \times 27$ & $14 \times 27$ & $14 \times 27$ & $14 \times 27$ & $20 \times 33$ & $20 \times 33$ & $20 \times 33$ & $20 \times 33$ \\
\hline Cen A & 6.4 & 0.3 & $<0.2$ & 2.0 & $<0.3$ & 9.8 & 1.2 & 22.3 & 54.5 & $\ldots$ & 1.4 \\
\hline Circinus & 35.2 & 1.2 & $<1.0$ & 21.8 & $<0.5$ & 67.9 & 5.9 & 93.2 & 151.0 & 1.5 & 4.0 \\
\hline M 51 & 1.0 & $\ldots$ & $\ldots$ & $\ldots$ & $\ldots$ & 1.9 & 1.0 & 4.6 & 9.6 & $\ldots$ & $\ldots$ \\
\hline M 87 & $<0.6$ & $\ldots$ & $\ldots$ & $\ldots$ & $\ldots$ & $<0.2$ & 0.5 & $<1.4$ & $<1.2$ & $\ldots$ & $\ldots$ \\
\hline Mkn 1 & $<0.8$ & $\ldots$ & $\ldots$ & $\ldots$ & $\ldots$ & 2.8 & $<0.4$ & $<1.0$ & $<1.3$ & $\ldots$ & $\ldots$ \\
\hline Mkn 3 & $\ldots$ & $\ldots$ & $\ldots$ & 3.4 & $\ldots$ & 12.6 & $\ldots$ & 3.0 & $\ldots$ & $\ldots$ & $<2.5$ \\
\hline Mkn 6 & $\ldots$ & $\ldots$ & $\ldots$ & $<0.5$ & $\ldots$ & $1.6^{3}$ & $\ldots$ & $<1.8$ & $\ldots$ & $\ldots$ & $\ldots$ \\
\hline Mkn 335 & $\ldots$ & $\ldots$ & $\ldots$ & $<0.3$ & $\ldots$ & 1.3 & $<0.3$ & $\ldots$ & $\ldots$ & $\ldots$ & $<0.5$ \\
\hline Mkn 463 & $<0.8$ & $\ldots$ & $\ldots$ & $\ldots$ & $\ldots$ & 4.3 & $\ldots$ & 1.2 & 2.0 & $\ldots$ & $\ldots$ \\
\hline Mkn 509 & $<1.5$ & $\ldots$ & $\ldots$ & $<0.6$ & $\ldots$ & 1.8 & $\ldots$ & $\ldots$ & $\ldots$ & $\ldots$ & $<2.0$ \\
\hline Mkn 573 & $\ldots$ & $\ldots$ & $\ldots$ & $\ldots$ & $\ldots$ & 7.9 & $\ldots$ & 2.9 & $\ldots$ & $\ldots$ & $\ldots$ \\
\hline Mkn 938 & $\ldots$ & $\ldots$ & $\ldots$ & $\ldots$ & $\ldots$ & $\ldots$ & $\ldots$ & $\ldots$ & $\ldots$ & $\ldots$ & $\ldots$ \\
\hline Mkn 1014 & $<0.6$ & $\ldots$ & $\ldots$ & $\ldots$ & $\ldots$ & $2.4^{3}$ & $<1.0$ & $<1.6$ & $<1.3$ & $\ldots$ & $\ldots$ \\
\hline NGC 613 & $\ldots$ & $\ldots$ & $\ldots$ & $<0.7$ & $\ldots$ & $\ldots$ & $\ldots$ & $\ldots$ & $\ldots$ & $\ldots$ & $<1.0$ \\
\hline NGC 1068 & 40.0 & $\ldots$ & $\ldots$ & 70.0 & $\ldots$ & 190.0 & $8.0^{3}$ & 55.0 & 91.0 & $<1.4$ & 18.0 \\
\hline NGC 1275 & $<1.3$ & $<0.5$ & $<0.4$ & $<0.4$ & $<0.5$ & $<0.5$ & $<0.4$ & $<1.7$ & 6.8 & $\ldots$ & $<1.0$ \\
\hline NGC 1365 & 13.5 & 0.8 & $<0.7$ & 3.9 & $<0.4$ & 14.6 & 1.8 & 36.1 & 73.8 & $\ldots$ & $<1.6$ \\
\hline NGC 3783 & 2.0 & $\ldots$ & $\ldots$ & $\ldots$ & $\ldots$ & 3.8 & $<0.5$ & $<2.0$ & 3.2 & $\ldots$ & $\ldots$ \\
\hline NGC 4051 & $\ldots$ & $\ldots$ & $\ldots$ & $\ldots$ & $\ldots$ & $\ldots$ & $\ldots$ & $\ldots$ & $\ldots$ & $\ldots$ & $\ldots$ \\
\hline NGC 4151 & 5.4 & $<0.2$ & $<0.4$ & 5.6 & $\ldots$ & 20.3 & 0.4 & 8.1 & 15.6 & $\ldots$ & 3.5 \\
\hline NGC 5506 & 3.0 & $<0.4$ & $<0.5$ & 2.4 & $\ldots$ & 13.5 & $0.6^{3}$ & 8.1 & 14.2 & $\ldots$ & $1.2^{3}$ \\
\hline NGC 5643 & 2.3 & $\ldots$ & $\ldots$ & $\ldots$ & $\ldots$ & 10.3 & $\ldots$ & 5.8 & 5.5 & $\ldots$ & $\ldots$ \\
\hline NGC 7469 & 9.2 & 0.9 & $<0.4$ & $0.6^{3}$ & $\ldots$ & 3.4 & 0.6 & 10.4 & 19.6 & $\ldots$ & $<1.0$ \\
\hline NGC 7582 & 5.2 & $<0.5$ & $<0.5$ & 3.1 & $\ldots$ & 11.6 & 0.8 & 11.3 & 21.8 & $<1.4$ & $1.0^{3}$ \\
\hline PKS 2048-57 & 2.2 & $\ldots$ & $\ldots$ & 2.7 & $\ldots$ & 8.5 & 0.5 & 2.7 & 3.5 & $\ldots$ & $<1.2$ \\
\hline TOL 0109-383 & $\ldots$ & $\ldots$ & $\ldots$ & 0.5 & $\ldots$ & 1.4 & $<0.3$ & $\ldots$ & $\ldots$ & $\ldots$ & $<2.0$ \\
\hline I Zw 1 & $<0.5$ & $\ldots$ & $\ldots$ & $\ldots$ & $\ldots$ & $<0.6$ & $\ldots$ & $<1.0$ & $<1.8$ & $\ldots$ & $<0.4$ \\
\hline I Zw 92 & $\ldots$ & $\ldots$ & $\ldots$ & $\ldots$ & $\ldots$ & 1.1 & $\ldots$ & 1.5 & $\ldots$ & $\ldots$ & $\ldots$ \\
\hline $3 \mathrm{C} 120$ & $\ldots$ & $\ldots$ & $\ldots$ & $\ldots$ & $\ldots$ & 7.5 & $<0.3$ & $<1.2$ & $\ldots$ & $\ldots$ & $\ldots$ \\
\hline
\end{tabular}

Table 3. Additional fine structure line fluxes and upper limits (in $10^{-20} \mathrm{~W} / \mathrm{cm}^{2}$ ).

\begin{tabular}{llllllllllll}
\hline \hline Galaxy & {$[\mathrm{Ca} \mathrm{IV}]$} & {$[\mathrm{Ca} \mathrm{VII}]$} & {$[\mathrm{Ca} \mathrm{V}]$} & {$[\mathrm{Ar} \mathrm{VI}]$} & {$[\mathrm{Si} \mathrm{VII}]$} & {$[\mathrm{Na} \mathrm{III}]$} & {$[\mathrm{Na} \mathrm{VI}]$} & {$[\mathrm{Ca} \mathrm{V}]$} & {$[\mathrm{Mg}$ V] } & {$[\mathrm{Fe} \mathrm{II}]$} & {$[\mathrm{Fe} \mathrm{III}]$} \\
\hline$\lambda_{\text {rest,vac }}(\mu \mathrm{m})$ & 3.207 & 4.087 & 4.158 & 4.530 & 6.492 & 7.318 & 8.611 & 11.482 & 13.521 & 24.519 & 33.038 \\
Resolution $^{3}$ & 125 & 150 & 140 & 135 & 215 & 180 & 150 & 110 & 185 & 220 & 200 \\
$E_{\text {ion }}(\mathrm{eV})^{1}$ & 50.9 & 108.8 & 67.3 & 75.0 & 205.1 & 47.3 & 138.4 & 67.3 & 109.2 & 7.9 & 16.2 \\
Aperture $^{2}$ & $14 \times 20$ & $14 \times 20$ & $14 \times 20$ & $14 \times 20$ & $14 \times 20$ & $14 \times 20$ & $14 \times 20$ & $14 \times 20$ & $14 \times 27$ & $14 \times 27$ & $20 \times 30$ \\
Circinus & $<0.9$ & $<3.5$ & $<3.0$ & 3.1 & $<1.0$ & $\ldots$ & $\ldots$ & $<0.8$ & $<1.5$ & $<0.5$ & $<5.0$ \\
NGC 1068 & 1.3 & $\ldots$ & $\ldots$ & 15.0 & $\ldots$ & 5.8 & $<16.0$ & $\ldots$ & $\ldots$ & $\ldots$ & $\ldots$ \\
\hline
\end{tabular}

${ }^{1}$ Lower ionization potential of the stage leading to the transition.

${ }^{2}$ In arcsec.

${ }^{3} \mathrm{In} \mathrm{km} \mathrm{s}^{-1}$.

structure lines), Table 3 (additional fine structure lines for few sources), and Table 4 (hydrogen recombination lines). The spectra and fluxes for NGC 4151 and NGC 1068 have already been published in Sturm et al. (1999) and Lutz et al. (2000b) respectively. We have repeated the fluxes (but not the spectra) here for convenience. Some of the Circinus data have been published in Moorwood et al. (1996), but later on more data have been taken. We have re-analyzed the old Circinus data with the newer calibration files in order to be consistent with the reduction of the additional observations. 
Table 4. Observed hydrogen recombination line fluxes (in $10^{-20} \mathrm{~W} / \mathrm{cm}^{2}$ ).

\begin{tabular}{llllll}
\hline \hline Galaxy & $\operatorname{Br} \beta$ & $\operatorname{Pf} \gamma$ & $\operatorname{Br} \alpha$ & $\operatorname{Pf} \beta$ & $\operatorname{Pf} \alpha$ \\
\hline$\lambda_{\text {rest,vac }}(\mu \mathrm{m})$ & 2.625 & 3.741 & 4.052 & 4.654 & 7.460 \\
Resolution $^{1}$ & 145 & 155 & 150 & 135 & 180 \\
Aperture $^{2}$ & $14 \times 20$ & $14 \times 20$ & $14 \times 20$ & $14 \times 20$ & $14 \times 20$ \\
Cen A & 0.9 & $\ldots$ & 0.8 & $\ldots$ & 0.2 \\
Circinus & 3.2 & $\ldots$ & 5.5 & $\ldots$ & 2.1 \\
M 51 & $<0.8$ & $\ldots$ & $\ldots$ & $\ldots$ & $\ldots$ \\
Mkn 335 & $<0.3$ & $\ldots$ & $\ldots$ & $\ldots$ & $\ldots$ \\
Mkn 463 & $<0.4$ & $\ldots$ & $\ldots$ & $\ldots$ & $\ldots$ \\
Mkn 1014 & $<0.3$ & $\ldots$ & $\ldots$ & $\ldots$ & $\ldots$ \\
NGC 1068 & 4.1 & $<0.4$ & 6.9 & $<10.0$ & $<3.0$ \\
NGC 1275 & $<0.2$ & $\ldots$ & $<2.2$ & $\ldots$ & $\ldots$ \\
NGC 1365 & 1.6 & $\ldots$ & 2.8 & $\ldots$ & 0.6 \\
NGC 3783 & $<0.8$ & $\ldots$ & $\ldots$ & $\ldots$ & $\ldots$ \\
NGC 4151 & 0.5 & $\ldots$ & $0.9^{3}$ & $\ldots$ & $\ldots$ \\
NGC 5506 & $0.7^{4}$ & $\ldots$ & 1.2 & $\ldots$ & $\ldots$ \\
NGC 5643 & $0.4^{5}$ & $\ldots$ & $\ldots$ & $\ldots$ & $\ldots$ \\
NGC 7469 & $0.75^{6}$ & $\ldots$ & $\ldots$ & $\ldots$ & $\ldots$ \\
NGC 7582 & 0.9 & $\ldots$ & 1.6 & $\ldots$ & $\ldots$ \\
PKS 2048-57 & $<0.5$ & $\ldots$ & $\ldots$ & $\ldots$ & $\ldots$ \\
TOL 0109-383 & $<0.5$ & $\ldots$ & $\ldots$ & $\ldots$ & $\ldots$ \\
I Zw 1 & $<0.4$ & $\ldots$ & $\ldots$ & $\ldots$ & $\ldots$ \\
\hline
\end{tabular}

\footnotetext{
${ }^{1} \mathrm{In} \mathrm{km} \mathrm{s}^{-1}$.

${ }^{2}$ In arcseconds.

${ }^{3}$ From $\operatorname{Br} \beta$ (case B, see Hummer \& Storey 1987).

${ }^{4}$ From $\operatorname{Br} \alpha$ (see note 3).

${ }^{5}$ From $\operatorname{Br} \gamma$ (Kowara et al. 1987, see note 3).

${ }^{6}$ from $\operatorname{Br} \gamma$ (Goldader et al. 1995, see note 3).
}

\section{Physical conditions in the NLR}

Diagnostics of the physical conditions in the Narrow Line Region are of interest given the wide range of possible excitation mechanisms and interpretations for its emission. These include models assuming ionization-bounded photoionization regions (e.g. T. Alexander et al. 1999, 2000), more complex photoionization models invoking a mixture of ionizationbounded and matter-bounded clouds (Binette et al. 1996, 1997) as well as shock excitation, for example, through jet-cloud interaction (e.g. Axon et al. 1998). Traditional optical diagnostics have been applied to large sets of objects but do have shortcomings. The [S II] $\lambda \lambda 6716,6731$ density diagnostic applies to low ionization species that may not be representative of the higher excitation regions and may be contaminated by nonAGN emission. The $[\mathrm{O}$ III] $(\lambda 4959+\lambda 5007) / \lambda 4363$ temperature diagnostic includes a weak and blended line that is notoriously difficult to measure. Combining infrared fine-structure lines with optical lines provides additional options. Given the large observing apertures for mid-IR instruments like ISOSWS, their use should be restricted mainly to high excitation species that are not contaminated by spatially extended starburst emission.

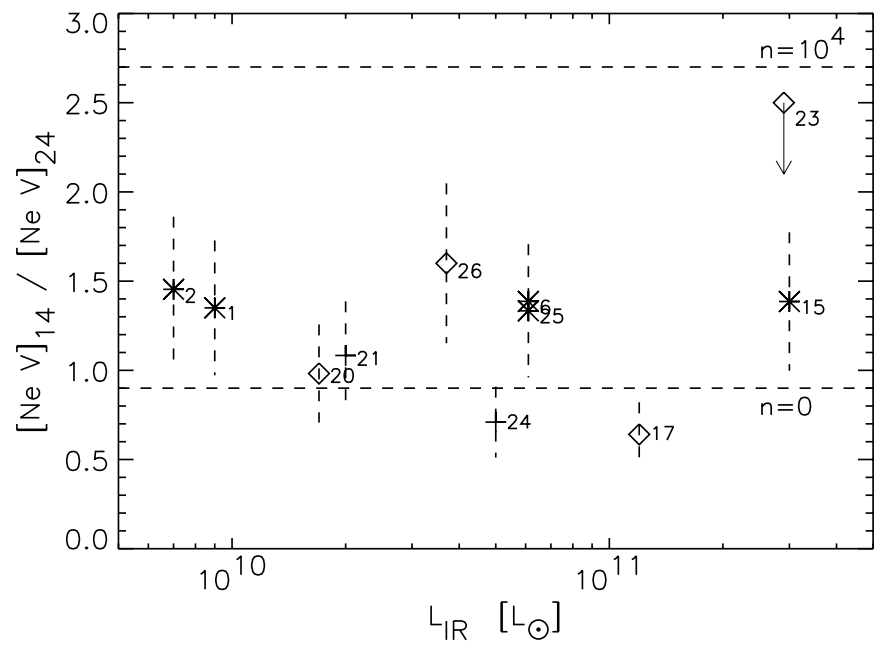

Fig. 1. The ratio of [Ne V] 14.3/24.3 $\mu \mathrm{m}$. Diamonds: Seyfert 1s. Stars: Seyfert 2s, Plus signs: NLXGs. The theoretical low density limit $(\approx 0.9)$ and the ratio for $n_{\mathrm{e}}=10^{4} \mathrm{~cm}^{-3}(2.7)$, for a temperature of $10000 \mathrm{~K}$, are shown as dashed lines. Error bars for a $20 \%$ line flux error are indicated. The numbering of galaxies can be found in the first column of Table 1 .

\subsection{Density}

Density-sensitive ratios of bright infrared fine-structure lines are observable for a range of ionization potentials. Because of possible contamination by starburst emission (cf. Lutz et al. $2000 \mathrm{~b}$ for the longer wavelength lines in NGC 1068), we do not discuss [S III] 18.71/33.48 $\mu \mathrm{m}$ and [Ne III] 15.55/36.01 $\mu \mathrm{m}$. The perhaps most interesting ratio is $[\mathrm{Ne} \mathrm{V}] 14.3 / 24.3 \mu \mathrm{m}$. These lines are not diluted by starburst contributions, since they have a lower ionization potential of $97 \mathrm{eV}$ and are undetected in ISO spectra of starburst galaxies (Genzel et al. 1998). Their ratio is largely insensitive to electron temperature and to extinction variations (see T. Alexander et al. 1999, their Fig. 3, for the theoretical $[\mathrm{Ne} \mathrm{V}]$ ratio as function of density).

All objects with measurable [Ne V] in our sample have $[\mathrm{Ne} \mathrm{V}]$ ratios indicating a low density $\left(n_{\mathrm{e}}=a\right.$ few 100 to a few $\left.1000 \mathrm{~cm}^{-3}\right)$, well below the critical densities $\left(\approx 5 \times 10^{4}\right.$ and $\left.5 \times 10^{5} \mathrm{~cm}^{-3}\right)$, see Fig. 1 . The average ratio is $1.1 \pm 0.4$ for the (4) Seyfert $1 \mathrm{~s}$, and $1.3 \pm 0.3$ for the (6) Seyfert 2 s, i.e. NLR densities for the two types agree for this fairly small sample, consistent with unification.

\subsection{Temperature}

Temperature diagnostics for photoionized gas invoke a combination of transitions that originate in levels separated by at least several $1000 \mathrm{~K}$. Combinations of ground state infrared fine-structure lines with optical/UV forbidden lines of the same ion provide maximum leverage for this task and represent excellent diagnostics provided practical requirements can be met: (1) Both IR and optical/UV lines have to be well observed, with decent $\mathrm{S} / \mathrm{N}$ and line-to-continuum. (2) Aperture corrections have to be understood - note the much larger ISO aperture compared to commonly used optical apertures. (3) Contamination by non-NLR emission has to be low. (4) Extinction corrections 


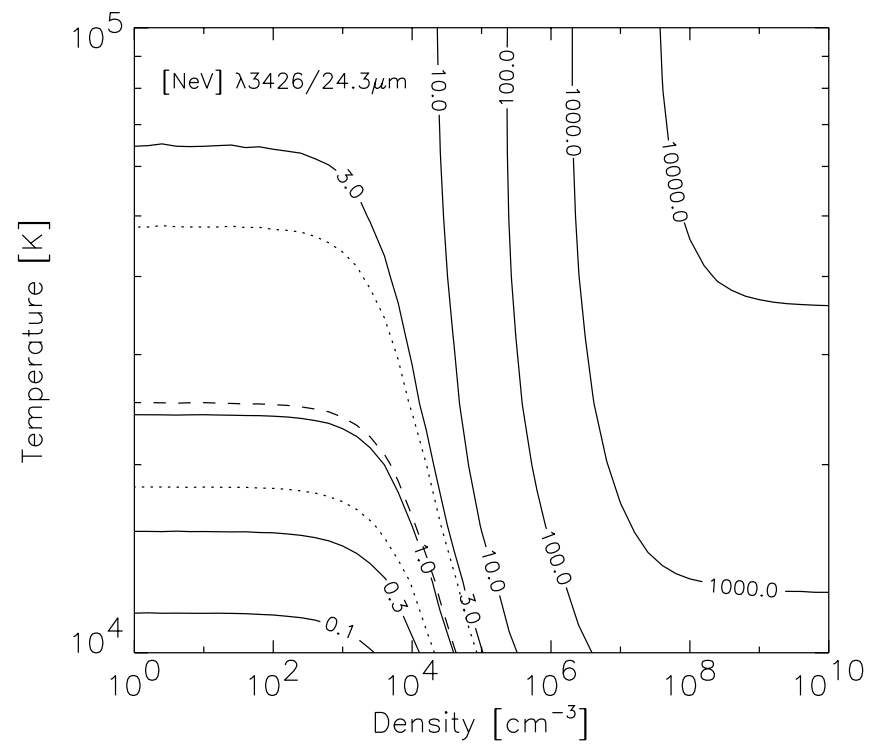

Fig. 2. Temperature-sensitive ratio of the $3426 \AA$ and $24.3 \mu \mathrm{m}$ lines of $[\mathrm{Ne} \mathrm{V}]$ as a function of electron density and temperature. The dashed line indicates the ratio for NGC 1068 and the dotted lines its uncertainty range considering measurement errors and uncertainty of extinction.

have to be understood. The last requirement is not trivial to meet. The extinction towards a NLR will not follow a simple foreground screen, as most evidently shown by the presence of intra-NLR dust (e.g. Cameron et al. 1993), and line profile variations between optical and infrared (cf. Lutz et al. 2000b for NGC 1068). Lines observed at different wavelengths may therefore not sample the same gas volume, even if fluxes are adjusted using simple extinction corrections. Hence we ignore combinations UV/IR (e.g. [OIV], [Mg V]) where these problems are most severe, and discuss a number of optical/IR diagnostics focussing on the well studied objects NGC 1068 and Circinus. Of particular interest are highly ionized regions not well sampled by the optical [O III] or [N II] temperature diagnostics.

One interesting species is [ $\mathrm{Ne} \mathrm{V}$ ] (lower ionisation potential $97 \mathrm{eV}$ ) which has very bright optical and mid-infrared transitions. Figure 2 shows the ratio predicted for the $3426 \AA$ and $24.3 \mu \mathrm{m}$ lines, using the atomic data of Lennon \& Burke (1994) and Nussbaumer \& Rusca (1979). The range of allowed values for NGC 1068 (combining our data with optical data of Marconi et al. 1996 and adopting $A_{V}=0.71$ ) is also indicated, adding a $20 \%$ measurement error for each line, and a $0.25 \mathrm{mag}$ uncertainty in $A_{V}$ to produce the maximum deviation. These uncertainties (particularly the one on extinction) lead to a wide range of electron temperatures ( $\sim 17000-45000 \mathrm{~K})$ being consistent with the NGC 1068 data for its density of $2000 \mathrm{~cm}^{-1}$.

Another high excitation species offers the potential of reducing the extinction effects. The brightest optical line of [Fe VII] is found at the longer wavelength of $6087 \AA$. With a lower ionisation potential of $99 \mathrm{eV}$, [Fe VII] samples a region similar to [Ne V]. The ratio of the optical [Fe VII] $6087 \AA$ line to the mid-infrared [Fe VII] lines is little sensitive to electron density in the regime determined above, and forms a
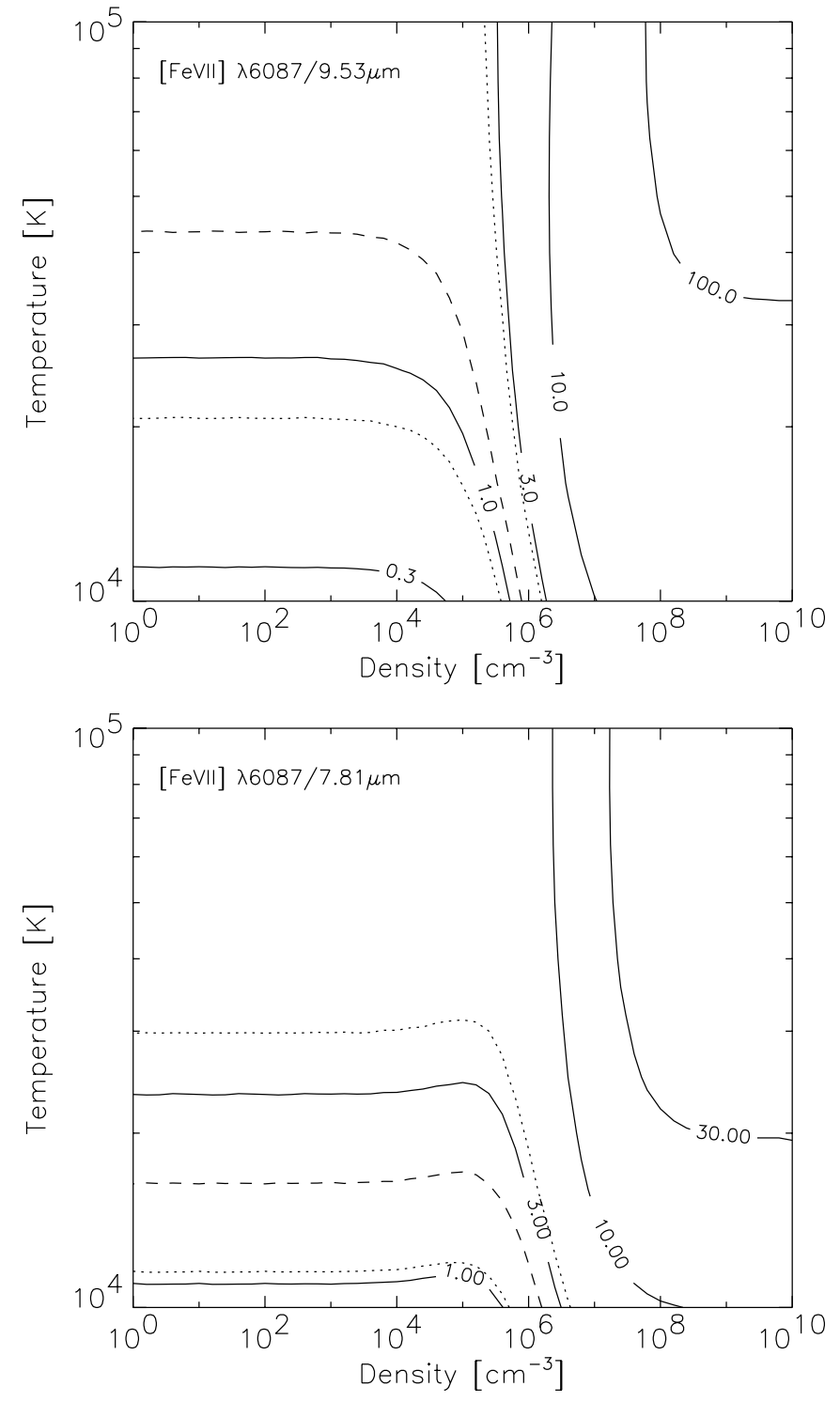

Fig. 3. Temperature-sensitive ratio of the $6087 \AA$ and the $9.53 \mu \mathrm{m}$ (top) and $7.81 \mu \mathrm{m}$ (bottom) lines of [Fe VII], as a function of electron density and temperature. The dashed line indicates the ratio for NGC 1068 and the dotted lines its uncertainty range considering measurement errors and uncertainty of extinction.

diagnostic of electron temperature in the NLR. Figure 3 shows the ratios from solving the rate equation using the atomic data of Berrington et al. (2000, and priv. communication ${ }^{1}$ ). We have observed the mid-IR [Fe VII] lines in NGC 1068 and Circinus. The extinction corrected line ratios for NGC 1068 lead to very different electron temperatures: $\approx 43000 \mathrm{~K}$ from $6087 \AA / 9.53 \mu \mathrm{m}$ but $\approx 16000 \mathrm{~K}$ from $6087 \AA / 7.81 \mu \mathrm{m}$. This discrepancy is also reflected in the extinction corrected ratio of the $9.53 \mu \mathrm{m}$ and $7.81 \mu \mathrm{m}$ lines which is 1.4 , significantly different from the $\approx 3-3.5$ expected for a wide range of conditions from the atomic data. For Circinus, the extinction corrected ratio of the $9.53 \mu \mathrm{m}$ and $7.81 \mu \mathrm{m}$ lines is similarly low, about 1.1. There is no immediate explanation for this inconsistency

${ }^{1}$ Collision strengths for $10000 \mathrm{~K}$ and corrections of three erroneous transition probabilities. 


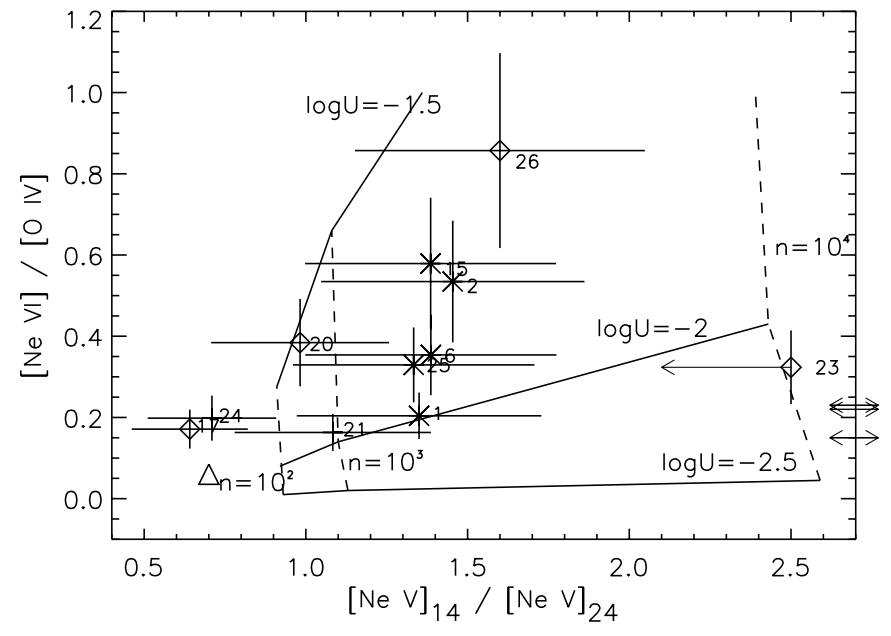

Fig. 4. Line ratio diagram of typical NLR tracers. Diamonds: Seyfert 1s. Stars: Seyfert 2s, Plus signs: NLXGs. Numbering of galaxies as in Fig. 1. The supernova remnant RCW103 (Oliva et al. 1999) is shown as a triangle. The $[\mathrm{Ne} \mathrm{VI]/[O} \mathrm{IV]} \mathrm{values} \mathrm{for} \mathrm{three} \mathrm{additional} \mathrm{sources}$ are indicated as horizontal arrows on the right hand $y$-axis (from top to bottom: 3C120, Mkn 509, Mkn 573). To constrain ionization parameter and density we have overplotted a model grid by Spinoglio et al. (2000) for a power law ionizing continuum of index $\alpha=-1.0$ : solid lines are $\log U=-2.5,-2$, and -1.5 , dashed lines are for $n=10^{2}$, $10^{3}$, and $10^{4}$.

between mid-IR FeVII fluxes that is observed independently in two sources. An explanation by extinction uncertainties is unlikely, since $\approx 10$ mag of additional visual extinction would be needed to selectively weaken the $9.53 \mu \mathrm{m}$ line which is inside the silicate feature. Uncertainties in the atomic data might be another possibility but are only partially supported by observations of the same lines in the planetary nebula NGC 7027: Salas et al. (2001) observe a ratio of 1.84 for the two mid-IR lines, closer to the value of 2.73 expected for the conditions in this nebula.

We summarize that, while there are indications for high (20000-30000 K) electron temperatures in the part of the narrow line region sampled by $E_{\text {ion }} \sim 100 \mathrm{eV}$ species, there is considerable uncertainty on extinction corrections and atomic data which prevents firm conclusions.

\subsection{Excitation}

Varying relative line strengths of low and high ionizaton lines, e.g. of the Neon sequence, seem to indicate a large variation in excitation among the galaxies in our sample. Line ratios from ions in different stages of excitation along with a photoionization model can be used to reconstruct the NLR radiation field produced by the central ionizing source. Mid-Infrared lines are particularly suited because they are little sensitive to extinction and electron temperature, and because they span a wide range of ionization potentials. In detailed studies we have analyzed three nearby Seyfert nuclei of our sample with elaborate photoionization models: Circinus (Seyfert 2 plus starburst), NGC 4151 (Seyfert 1.5) and NGC 1068 (Seyfert 2), see Moorwood et al. (1996), and T. Alexander et al. (1999, 2000).
These models were able to reconstruct the intrinsic spectral energy distribution (SED) of the ionizing source in the extreme UV, where a "Big UV Bump" around $100 \mathrm{eV}$ is expected as a signature from a hot thin accretion disk. The results are consistent with such a "Big UV Bump", but also suggest for some sources of both types (Seyfert 1 and 2) the presence of neutral absorbers between the AGN's extreme ultraviolet emitting source and the NLR. Such (UV) absorbers have been suggested independently by studies of UV absorption lines (Kriss et al. 1992; Kraemer et al. 1999, 2001). This detailed photoionization modeling requires a large number of mid-IR lines (of good $\mathrm{S} / \mathrm{N}$ ) supplemented with UV/optical/near-IR lines. For most of the galaxies in our sample the compilation of lines from ISO and the literature is not as complete as for the three examples given above. Hence, we do not attempt a similar modeling for them. Instead, we try to constrain the NLR excitation by directly comparing our observations to standard photoionization models from the literature.

The main input parameters for photoionization models are the electron density $n_{\mathrm{e}}$ and the ionization parameter $U$, i.e. the number of ionizing photons per hydrogen atom at the inner face of the ionized cloud. The ratio of lines from ions of similar ionization potential but with different critical densities (e.g. the [Ne V] 14/24 ratio) are good tracers of the electron densities (see Sect. 5.1). Vice versa, the ratio of lines with similar critical density but from ions of different ionization potential (like $[\mathrm{Ne} \mathrm{VI}] /[\mathrm{OIV}]$ ) is sensitive to the ionization parameter. In Fig. 4 we have used this to construct a diagram to constrain $n_{\mathrm{e}}$ and $U$. We have chosen the lines of [Ne V], [Ne VI] and [O IV] because they are not affected by photoionization by stars and because they are generally among the brightest high ionization lines. For comparison we show in Fig. 4 the location of the supernova remnant RCW103 (Oliva et al. 1999), as an example for a strong shock source, and a photoionization model grid taken from Spinoglio et al. (2000). This model grid was computed for a power law with an ionizing continuum of in$\operatorname{dex} \alpha=-1.0$ and for various ionization parameters and electron densities. We draw two conclusions from this comparison: firstly, all galaxies are consistent with these standard photoionization models, with average ionization parameters $\log U$ between -1.5 and -2 and (as seen already in Sect. 5.1) average densities between a few 100 and a few $1000 \mathrm{~cm}^{-3}$. Such a simple comparison can not, however, distinguish between simple power laws and more complex "Big UV Bump" models. As noted earlier, the detailed modeling of single sources required for such an analysis is outside the scope of this paper. Secondly, within our (small) sample, we do not see significant differences between the AGN sub-types.

\subsection{NLR line profiles}

Structures and velocity fields in the NLR can be studied by an analysis of line profiles. Numerous studies of the emission line profiles in Seyfert galaxies have shown that in many cases the lines exhibit blueward asymmetries and are blueshifted with respect to the galaxies systemic velocity. The common interpretation for these profile asymmetries is that they are caused 
Table 5. Classes of line profile asymmetries.

\begin{tabular}{|c|c|c|c|c|}
\hline & Class I & Class II & Class IIIa & Class IIIb \\
\hline $\begin{array}{l}\text { optical [O III] profile } \\
\text { MIR [O IV] profile } \\
\text { examples }\end{array}$ & $\begin{array}{l}\text { symmetric } \\
\text { symmetric, = optical } \\
\text { 3C } 120^{1}, \text { PKS } 2048^{2}\end{array}$ & $\begin{array}{l}\text { asymmetric } \\
\text { symmetric } \\
\text { Mkn } 509^{2} \text {, NGC } 5643^{2}\end{array}$ & $\begin{array}{l}\text { asymmetric } \\
\text { asymmetric, = optical } \\
\text { NGC } 3783^{2} \text {,NGC } 4151{ }^{3}{ }^{3} \\
\text { NGC } 7469^{1} \text {, To } 10109^{2}\end{array}$ & $\begin{array}{l}\text { asymmetric } \\
\text { asym./non-gauss., } \neq \text { optical } \\
\text { NGC } 1068^{3} \text {,Mkn } 1^{1}, \text { Mkn } 3^{3} \text {, } \\
\text { Mkn } 463^{2}, \text { NGC } 5506^{3}\end{array}$ \\
\hline
\end{tabular}

${ }^{1}$ Optical line profile from Vrtilek \& Carleton (1985).

${ }^{2}$ Optical line profile from Whittle (1985).

${ }^{3}$ Optical line profile from Veilleux (1991).
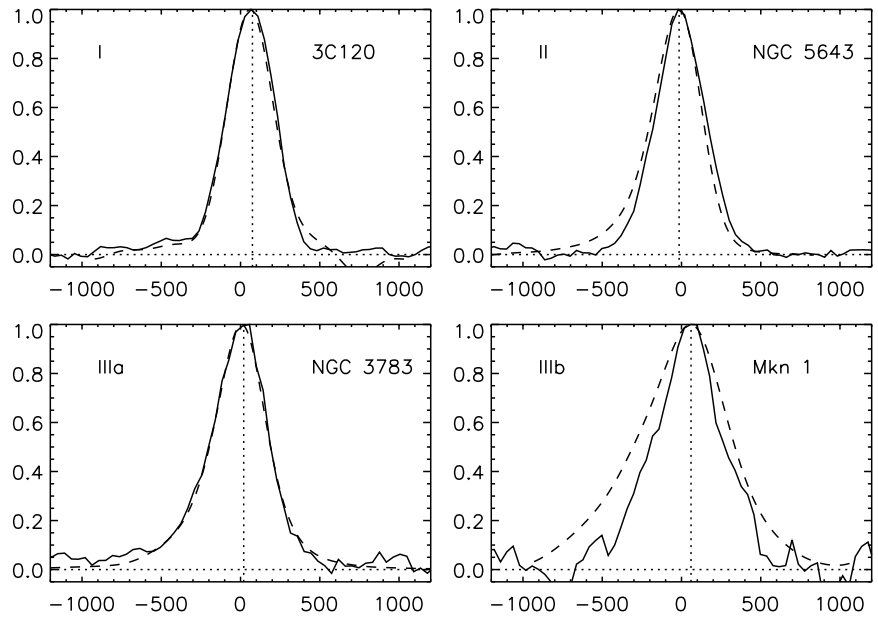

Fig. 5. Comparisons between optical and MIR line profiles (continuum subtracted and normalized to peak flux density), for four different cases as discussed in the text. Solid lines show the MIR [O IV] $26 \mu \mathrm{m}$ line, taken from the ISO-SWS data set. Dashed lines are optical [O III] $5007 \AA$ lines, smoothed to the SWS resolution. References for the optical line profiles are given in Table 5 . The velocity scale on the $x$-axis (in $\left[\mathrm{km} \mathrm{s}^{-1}\right]$ ) is relative to the systemic velocities given in Table 1.

by differential extinction in an outflow or inflow of clouds with a modest amount of mixed-in dust. Observations in the infrared can obviously test these scenarios, because infrared lines suffer more than an order of magnitude less extinction than in the optical. Hence, they should not or only marginally show asymmetries. Sturm et al. (1999) and Lutz et al. (2000b) have presented the first such studies of optical-to-mid-IR line profile comparisons for NGC 4151 and NGC 1068. In these two sources the outflow-plus-dust scenario seems to be (at least partially) wrong, since the MIR lines show asymmetries similar to their optical counterparts.

Optical [O III] line profile information exists for many objects in our data set. These can be used for a comparison with the MIR [O IV] line, as described in Sturm et al. (1999). We have convolved the optical line profiles with the SWS instrumental profile (Gauss profiles with FWHM as given in Table 2) in order to smooth these profiles to the resolution of SWS. Four such examples of an optical-MIR comparison are shown in Fig. 5. The objects in our sample can be grouped roughly into three different classes in terms of line profile asymmetries and agreement of MIR lines with their optical counterparts, as summarized in Table 5. In some cases (Class I) the optical line profile of [O III] is quite symmetric and of Gaussian shape. In these cases, not surprisingly, the MIR and optical line profiles match each other very well. Another group of objects (Class II) shows strong blueward asymmetries in the optical, while the MIR lines are rather symmetric. This is exactly what scenarios with infall our outflow of NLR clouds with mixed-in dust predict. We only found two such cases in our sample. The third class of objects has asymmetric optical lines, but MIR lines which are inconsistent with these scenarios. This class can be further divided into two sub-classes: in Class IIIa the MIR lines are asymmetric, too, and agree well with the optical lines. This case has been studied in more detail in an analysis of NGC 4151 by Sturm et al. (1999), and can be explained, for instance, by a true asymmetry in the distribution of the NLR clouds, or, in the case of NGC 4151, by a central, optically very thick, but geometrically thin absorber on parsec scales. In Class IIIb the NIR lines are asymmetric (or symmetric but with non-Gaussian profiles), but different from their optical counterparts. One member of this class is NGC 1068, which has been analyzed by Lutz et al. (2000b). This suggests that parts of the NLR are significantly obscured in the optical, but not enough to also block the MIR lines. Similar to Class IIIa, the remaining MIR profile asymmetries may be either due to an intrinsic asymmetry of the NLR, or due to a very high density obscuring component which is hiding part of the NLR even from infrared view. We note that for some of the objects with MIR lines of good S/N (Cen A, M 51, MKN 573, NGC 1365, and NGC 7582) there is, to our knowledge, no (suitable) optical line profile information available in the literature.

Many objects in our sample exhibit differences in the profiles of lines with different ionization potential. For instance, the Circinus galaxy shows symmetric low ionization lines, but asymmetric high ionization lines with the typical blueward asymmetries. Vice versa, in NGC 7582 high ionization lines are very symmetric, while the low ionization lines are strongly asymmetric. Correlations, as well as anti-correlations, of line asymmetries with critical density and/or ionization potential have been claimed in many publications. For NGC 7582 a contribution to the low ionization lines from a starburst component with asymmetric spatial distribution could be an additional/alternative solution. For the Circinus galaxy the situation is even more complex, since Oliva et al. (1994) reported asymmetric low ionization lines. Finally, for some objects in our sample all MIR line profiles appear to be quite similar. 


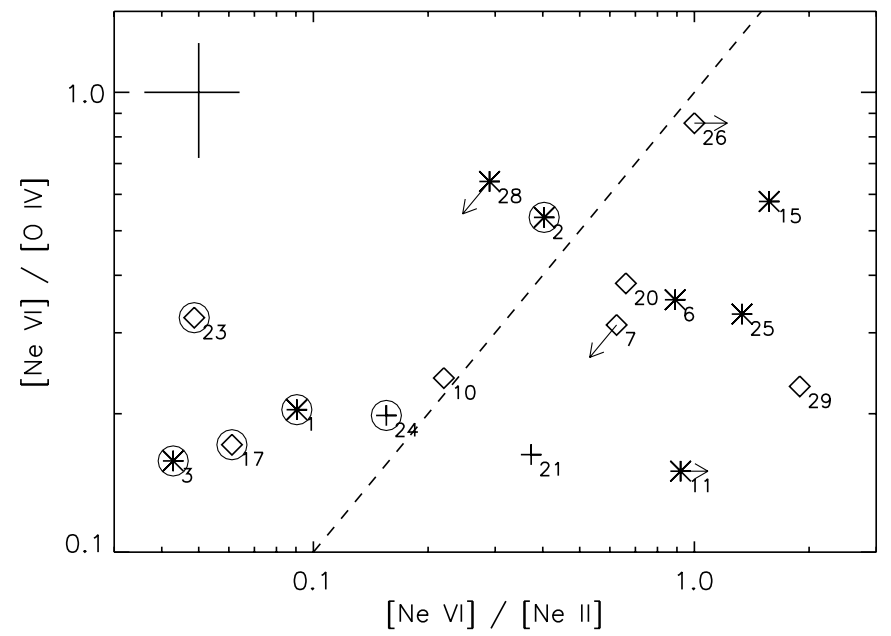

Fig. 6. The ratio $[\mathrm{Ne} \mathrm{VI}] /[\mathrm{O} I V]$ vs. [Ne VI]/[Ne II]. Same symbols and numbering as in Fig. 1. A typical error bar (according to a $20 \%$ individual line flux error) is shown in the upper left corner. An empirical dividing line is drawn as dashed line. Composite sources according to their PAH spectra are encircled. For galaxies \#26, 28 and 29 no ISO PAH spectra exist.

For instance, the profiles of Cen A, M 51, MKN 573, NGC 1365 (for which no optical counterparts exist) are quite symmetric and of Gaussian shape, regardless of ionization potential or critical density.

It appears that there is no unique answer to what causes the line profile asymmetries in Seyfert galaxies. Extinction by dust on different spatial scales and with varying column densities, sub-structure and true asymmetries in the spatial distribution of NLR clouds may all play a role with varying degrees of importance. For some objects the assignment to a certain group is not unique and depends also on $\mathrm{S} / \mathrm{N}$. We use this classification purely to obtain an overview of this complex issue. We refrain from a more detailed analysis of line profiles in this paper because it requires a careful study of the effects of aperture differences (spatial resolutions) between the optical and MIR observations, and, in many cases, a larger data set of high ionization lines with good $\mathrm{S} / \mathrm{N}$.

\section{Composite sources}

As discussed in Sects. 1 and 2 for many active galaxies an additional contribution from star forming regions within the SWS apertures is to be expected. In these cases ("composite sources") some fraction of the measured fluxes of low lying fine structure lines (excitation potential $\leq 50 \mathrm{eV}$ ) will be produced by photoionization from stars rather than from the AGN. In the previous sections we have therefore concentrated on high excitation lines ([O IV], [Ne V], [Ne VI], [Fe VII]) with little or no contamination from possible starburst components. However, for our considerations in the following sections low ionization lines like [Ne II] will be important. Hence, an independent identification of composite sources is desirable.

One such independent indicator is the strength of PAH features in the 5 to $12 \mu \mathrm{m}$ range, which are known to be strong in star forming regions, but more or less absent in the spectra

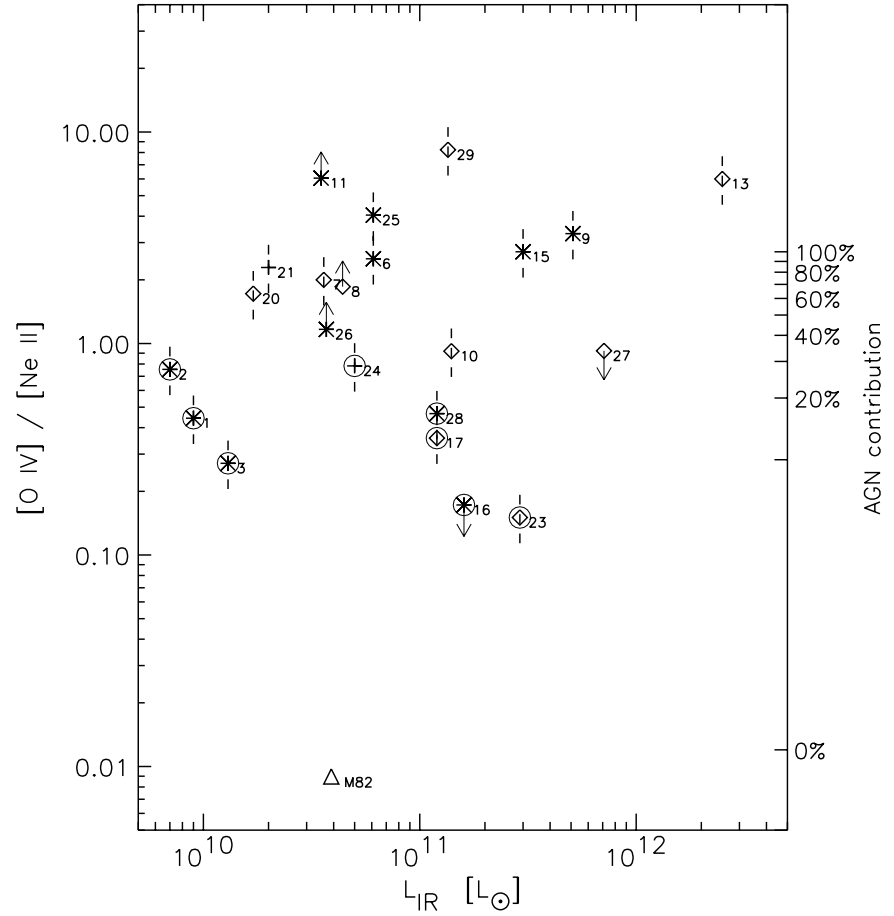

Fig. 7. The ratio [O IV] $25.9 \mu \mathrm{m} /[\mathrm{Ne}$ II] $12.8 \mu \mathrm{m}$. Same symbols and numbering as in Fig. 1. Composite objects are encircled. The starburst prototype M 82 is shown as a triangle. Denoted on the right hand $y$ axis is a simple linear "mixing" model of AGN contribution to the bolometric luminosity, in percent (see text). The [O IV]/[Ne II] ratios for $100 \% \mathrm{AGN}$ and $0 \% \mathrm{AGN}$ (=100\% starburst) are assumed to be 2.7 and 0.012 respectively.

of pure AGNs. Genzel et al. (1998), Rigopoulou et al. (1999), and Tran et al. (2001) have analysed a large number of low resolution ISOPHOT-S, ISOCAM-CVF and SWS spectra and used the line-to-continuum $(\mathrm{L} / \mathrm{C})$ ratio of the $7.7 \mu \mathrm{m}$ PAH feature to distinguish between starburst and AGN dominated sources. Following their method (and using the ISO archive as well as the spectra published in Rigopoulou et al. 1999; Spoon et al. 2002) we classify Cen A, Circinus, M 51, NGC 613, NGC 1365, NGC 7469, and NGC 7582, i.e. those sources with a $7.7 \mu \mathrm{m} \mathrm{L/C}$ ratio greater than 1 , as composite sources. As a consistency check, and for the cases where no PAH spectrum is available (Tol 0109, I Zw 92, and 3C 120), we have plotted in Fig. 6 the position of the targets in a diagram of [Ne VI]/[O IV] versus $[\mathrm{Ne} \mathrm{VI}] /[\mathrm{Ne} \mathrm{II}]$. As discussed in Sect. 5.3, the ratio $[\mathrm{Ne} \mathrm{VI}] /[\mathrm{OIV}]$ traces the AGN excitation without contamination from a possible star formation component. [Ne VI]/[Ne II] on the other hand, which is also an excitation indicator, can be influenced by contributions from star forming regions to the $[\mathrm{Ne}$ II] line. In such a diagram one expects the pure AGNs to lie roughly along a diagonal (both ratios increase with increasing hardness of the radiation). Composite sources, however, will have relatively stronger [Ne II] lines and should lie in a region further to the left than the pure AGN sources. In fact, all sources with known substantial starburst contributions, i.e. with strong PAH features, fall onto the left part of the diagram (Cen A, Circinus, M 51, NGC 1365, NGC 7469, and NGC 7582, marked with an enclosing circle in Fig. 6). 


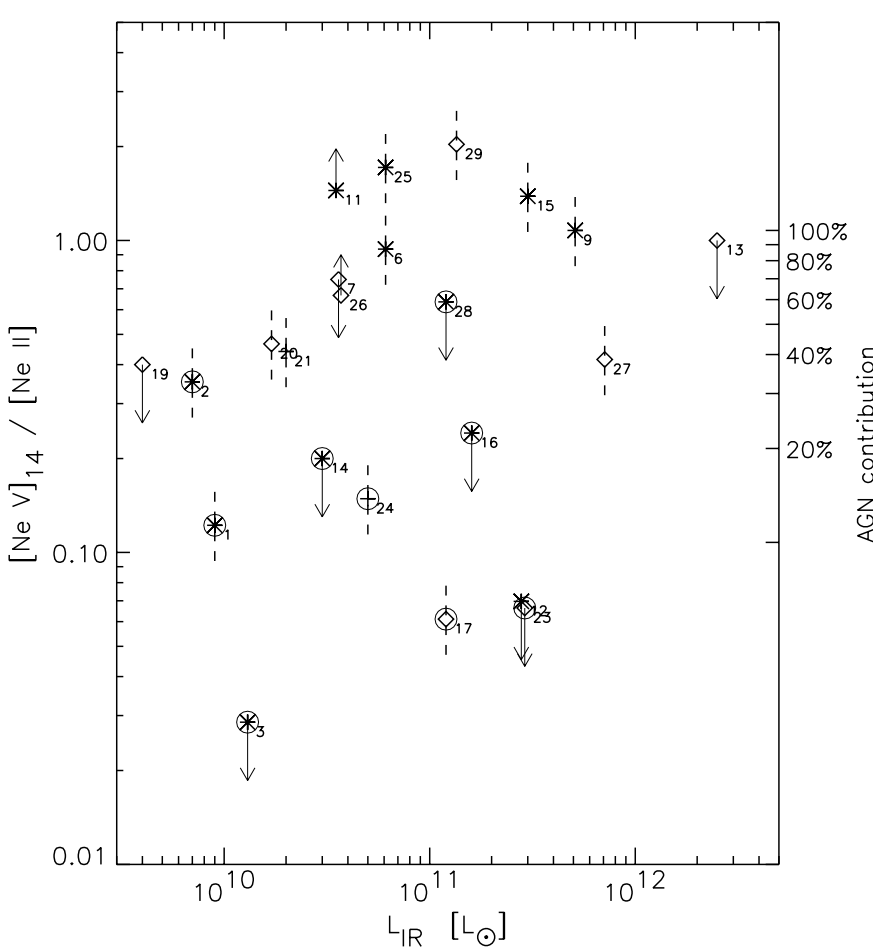

Fig. 8. The ratio [Ne V]14.3 $\mu \mathrm{m} /[\mathrm{Ne}$ II] $12.8 \mu \mathrm{m}$. Same symbols and numbering as in Fig. 1. Composite objects are encircled. Denoted on the right hand $y$-axis is a simple linear "mixing" model of AGN contribution to the bolometric luminosity, in percent (see text). The $[\mathrm{Ne} \mathrm{V}] /[\mathrm{Ne} \mathrm{II}]$ ratios for $100 \% \mathrm{AGN}$ and $0 \% \mathrm{AGN}(=100 \%$ starburst) are assumed to be $=1.1$ and 0 respectively.

Pure AGNs (like NGC 4151 and NGC 1068) are located in the right hand part. In Fig. 6 we have indicated an empirical dividing line (dashed). For simplicity we have chosen the line where [O IV] equals [Ne II]. According to this diagram one additional source without (good S/N) PAH data, I Zw 92 (\#28), has to be classified as composite source. This classification is confirmed by independent UV to near-IR observations (Heckman et al. 1997; Gonzalez Delgardo et al. 2001). We consider Mkn 509 (\#10) a borderline case. Another source, NGC 1275, can not be shown at its exact position in the diagram, but according to the upper limits for [O IV] and [Ne VI] (and the measured value for $[\mathrm{Ne} \mathrm{II}]$ ) the position must be in the composite source region. The galaxies that we classify as composite sources according to this scheme are indicated in Table 1 as "Sy + SB". The location of composite sources (as indicated by strong PAH features) in the left part of Fig. 6 suggests that a small ratio of $[\mathrm{NeVI}]$ and $[\mathrm{NeII}](\leq 0.1)$ is an independent empirical indicator for a composite object.

\section{New mid-IR diagnostic diagrams}

Deep surveys carried out by future infrared missions (such as SIRTF, SOFIA, ASTRO-F, or Herschel) will sample infrared bright galaxies over a wide range of redshifts and luminosities. Quantitative spectroscopy of mid-infrared emission lines will be an important diagnostic tool for determining the detailed properties of distant, dusty galaxies, the source of the extragalactic background, and the origin of nuclear activity in

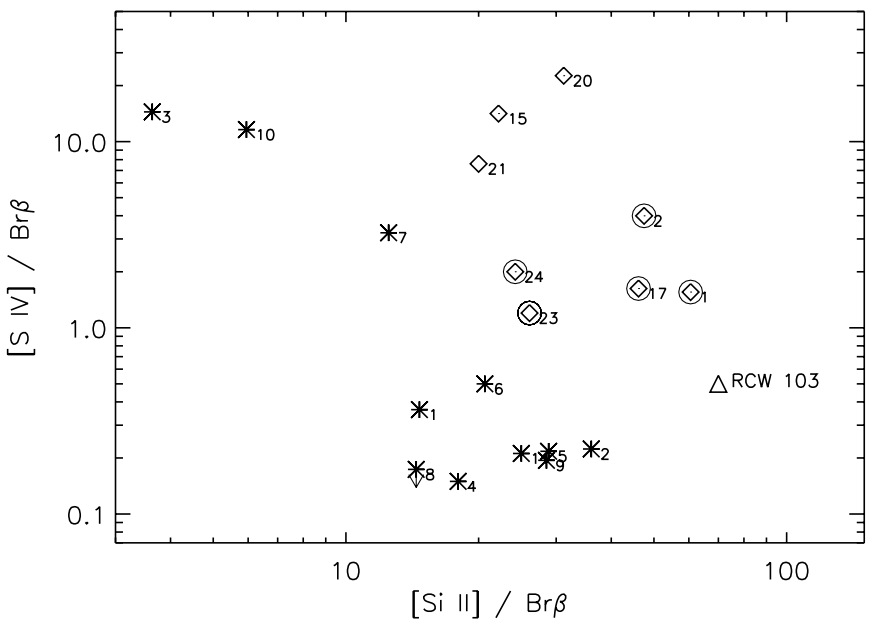

Fig. 9. A MIR diagnostic diagram to distinguish starbursts and AGNs in dusty galaxies - here: [S IV] $10 \mu \mathrm{m} / \mathrm{Br} \beta$ versus [Si II] $34 \mu \mathrm{m} / \mathrm{Br} \beta$. AGNs are shown as diamonds, starburst galaxies (Verma et al., in prep.) as stars. The shock source RCW 103 is shown as triangle. Composite objects are encircled. For three AGNs $\operatorname{Br} \beta$ was calculated from other Brackett lines (see Table 4). The numbering of the AGNs is taken from Table 1, for the starbursts it is as follows: 1: M 82, 2: IC 342, 3: IIZw 40, 4: NGC 253, 5: NGC 3256, 6: NGC 3690A, 7: NGC 4038/39, 8: NGC 4945, 9: NGC 5236, 10: NGC 5253, 11 : NGC 7552.

galaxies. Many of the fundamental questions of galaxy formation and evolution depend substantially on the fraction of the total energy output of distant sources that is produced by star formation rather than AGN activity. A large energy contribution from hidden AGNs would complicate the deduction of the star formation history of the Universe from galaxy luminosity functions. In the past, optical line ratios have been used as a tool for distinguishing between the different possible excitation mechanisms and energy sources in galaxies (e.g. Baldwin et al. 1981; Veilleux \& Osterbrock 1987). This works very well for objects with dust extinction less than $A_{V} \sim 5$, but becomes unreliable for heavily obscured objects (see, e.g., Veilleux et al. 1995; Veilleux et al. 1999). Hence, mid-infrared analogues of the classical optical diagrams are highly desirable. Genzel et al. (1998) presented a first empirical version of such a mid-IR tool, a diagram with the flux ratio of a high to a low excitation line $([\mathrm{OIV}] /[\mathrm{Ne} I I]$, or $[\mathrm{Ne} \mathrm{V}] /[\mathrm{Ne} I I])$ on one axis together with the AIB (PAH) strength on the other axis. Their finding that the $[\mathrm{OIV}] /[\mathrm{Ne} \mathrm{II}]$ ratio is much higher in AGNs than in starbursts can now be confirmed on a broader statistical basis. In starburst galaxies this ratio reaches values of a few times 0.01 at most (Lutz et al. 1998), whereas in the AGNs of our sample it does not drop below 0.1 (1.0 for pure AGNs), as shown in Fig. 7. For comparison we show in Fig. 8 the ratio of $[\mathrm{Ne} \mathrm{V}] 14 \mu \mathrm{m} /[\mathrm{Ne}$ II] . Those active galaxies in our sample with the lowest ratios in Figs. 7 and 8 are the ones we have identified earlier as composite sources (encircled in the figures), i.e. AGNs with a significant contribution from star formation to their spectra. On the right hand $y$-axes of Figs. 7 and 8 we have denoted a simple linear "mixing" model to read off the AGN contribution to the bolometric luminosity. This model will be further discussed in Sect. 8. 


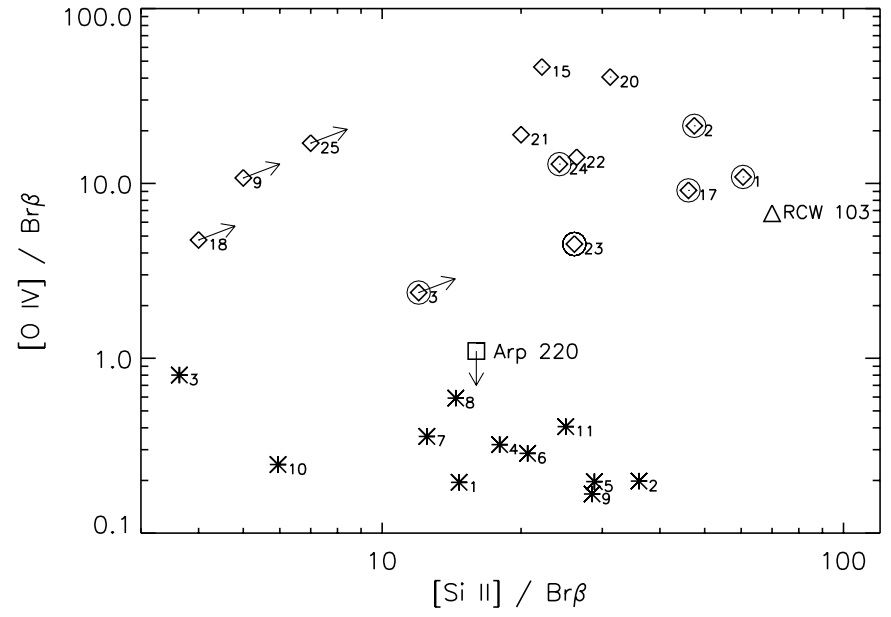

Fig. 10. Another example of MIR diagnostic diagrams - here: [OIV] $26 \mu \mathrm{m} / \operatorname{Br} \beta$ versus [Si II] $34 \mu \mathrm{m} / \operatorname{Br} \beta$. Symbols and numbering as in Fig. 9. The ULIRG Arp 220 (Sturm et al. 1996) is shown as a square, the SN remnant RCW 103 (Oliva et al. 1999) as triangle.

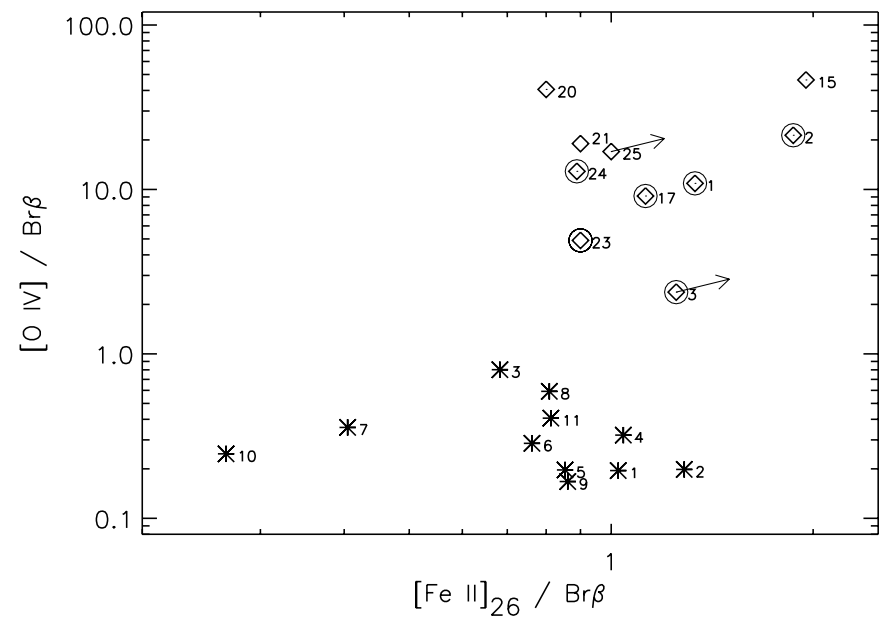

Fig. 11. Another example of MIR diagnostic diagrams - here: [OIV] $26 \mu \mathrm{m} / \mathrm{Br} \beta$ versus [Fe II] $26 \mu \mathrm{m} / \mathrm{Br} \beta$. Symbols and numbering as in Fig. 9.

Perhaps the best optical discriminator between photoionization by power-law spectra and by OB stars is the diagram in Fig. 3 of Veilleux \& Osterbrock (1987). It employs a moderately ionized line ([O III]) on one axis and a line of [OI], normalized to hydrogen recombination lines. Our data set allows the construction of MIR analogues of this "VO diagram" which are much less prone to extinction. We have selected [S IV]10.5 $\mu \mathrm{m}$ instead of [O III], which has an almost identical ionization potential, [Si II] $34 \mu \mathrm{m}$ instead of [O I], i.e. a line with an ionization potential below $13.6 \mathrm{eV}$, and $\operatorname{Br} \beta$ for normalization. For comparison we have used the ISO-SWS starburst data set of Verma et al. (in prep.). Starbursts and AGNs are clearly separated in this diagram (Fig. 9), not only because [S IV] is stronger in AGNs than in normal starbursts, but also because [Si II] is (on average) stronger, due to the higher importance of partially ionized zones in AGNs. Low metallicity starbursts, like IIZw40 and NGC 5253, have much harder radiation fields than normal starburst galaxies. In these cases [S IV] can be as strong as in AGNs, but [Si II] is much weaker. The overall appearance of Fig. 9 is thus very similar to the optical VO diagram. In contrast to its optical counterpart our mid-IR version also includes Seyfert 1 galaxies, since we did not detect any broad line components in the $\operatorname{Br} \beta$ lines. For a complete comparison of the optical and mid-IR diagrams our data set is missing galaxies of the LINER type. Their position in the midIR diagram is expected in the lower right corner, but remains to be tested in future infrared missions.

Several other versions of mid-IR diagnostic diagrams (with lines at different wavelengths) are similarly well suited for a distinction of excitation mechanisms. This allows to cover different redshift ranges or to adjust the method to the wavelength coverage of different detectors. For instance, it is possible to replace [S IV] $10.5 \mu \mathrm{m}$ with [O IV] $26 \mu \mathrm{m}$, and [Si II] $34 \mu \mathrm{m}$ with [Fe II]26 $\mu \mathrm{m}$ (Figs. 10 and 11). Similar diagnostic diagrams, based on theoretical modelling, with different sets of (mostly weaker) lines have been proposed by Spinoglio \& Malkan (1992) and Voit (1992). In contrast to the optical VO diagrams, the mid-IR versions can be applied to dusty systems with much higher extinctions, such as Ultraluminous Infrared Galaxies (ULIRGs). ULIRGs are believed to be local analogues of those distant dusty galaxies, which are (and will be) found in great numbers in deep infrared galaxy surveys. As discussed above, the identification of their energy source will be a major task for future infrared missions. In Fig. 10 we show the position of the well known ULIRG Arp 220 (Sturm et al. 1996). Its position in the diagram is consistent with predominant powering by intense star formation, which is in accordance with earlier studies (e.g. Sturm et al. 1996; Genzel et al. 1998).

\section{The source of the far-infrared luminosity}

In the previous section we have presented tools to detect AGNs in dusty galaxies. The presence of an AGN, however, does not automatically imply that the AGN activity is the dominant energy source in these galaxies. A significant fraction of the bolometric luminosity of Seyfert galaxies is emitted in the mid and far infrared by thermal (dust) emission. For a quantitative understanding of the contribution from AGNs to the total energy output of distant galaxies it is therefore essential to know whether the source powering the IR emission is (re-)radiated light from the AGN or from distributed star formation in the host galaxy.

In the following we will compare luminosities in different tracers for Seyferts and Starbursts in an attempt to shed light on these issues. We have selected [O IV] (as a high excitation line), [Ne II] (as a low excitation line), and the mid- and farinfrared continua. In all cases, we will keep track of the composite AGNs already identified in Sect. 6.

The mid-IR $(\lambda<40 \mu \mathrm{m})$ emission of AGNs is dominated by dust continuum re-radiating energy from black hole accretion. Orientation effects in a unified scheme cause variations in its level between Seyfert types 1 and 2, and extended star formation can contribute, more visibly in Sy2s with their lower AGN continuum (Clavel et al. 2000). In contrast, the origin 


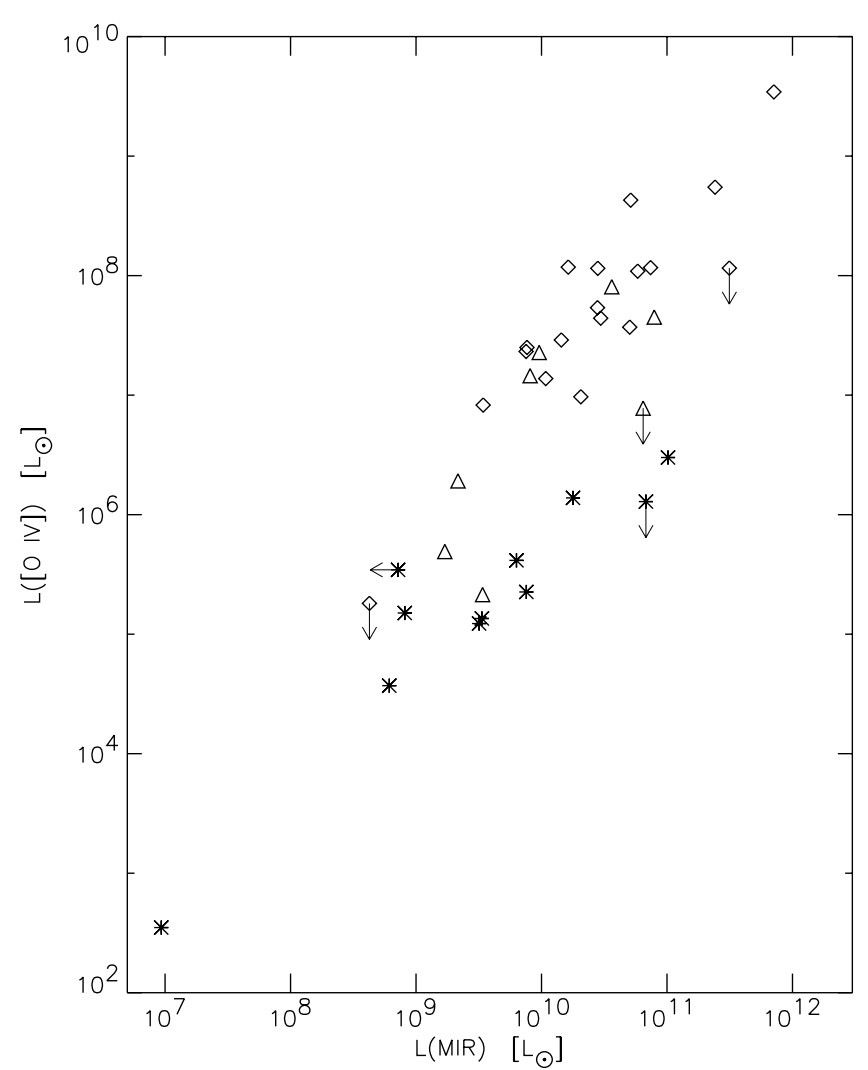

Fig. 12. [OIV] luminosity vs. MIR luminosity for AGNs (diamonds) and starburst galaxies (stars). Composite sources (see text) are indicated as triangles.

of the $40-120 \mu \mathrm{m}$ far-infrared emission of AGNs is considerably uncertain. Both the AGN proper and star formation have been advocated as energy source in the literature (cf. the review by Genzel \& Cesarsky 2000 and references therein). For starbursts, the mid-IR emission will be dominated by the aromatic emission features and transiently heated small grains. The farIR emission originates in large dust grains, and all are heated by newly formed or older stars (e.g. Desert et al. 1990).

[O IV] originates purely from the NLR in AGNs. In a unified scheme, the NLR line luminosity should be orientation independent and a good tracer of AGN power, in particular when using an extinction insensitive and modest excitation line like [OIV]. The much fainter starburst [OIV] emission relates to shocks or some very hot stars (Lutz et al. 1998). [Ne II] is a pure and fairly good tracer of the hot star emission in starbursts. In AGNs the [Ne II] from the NLR is more easily contaminated by starburst emission than the higher excitation [OIV] line.

We have computed mid-IR $(12+25 \mu \mathrm{m})$ and far-IR $(60+100 \mu \mathrm{m})$ band luminosities from the respective IRAS fluxes following Sanders \& Mirabel (1996), using $F_{\text {MIR }}=$ $1.26 \times\left(5.16 S_{25}+13.48 S_{12}\right) \times 10^{-18} \mathrm{~W} / \mathrm{cm}^{2}$, and $F_{\mathrm{FIR}}=1.26 \times$ $\left(S_{100}+2.58 S_{60}\right) \times 10^{-18} \mathrm{~W} / \mathrm{cm}^{2}$, where $\mathrm{S}$ is the flux density in Jy (from IRAS FSC), and $L=4 \pi D^{2} F=3.132 \times 10^{4} \times D_{\mathrm{Mpc}}^{2} F L_{\odot}$. Our results are summarized in Figs. 12-15, showing correlations of [O IV] and [Ne II] line luminosities with MIR and FIR continuum luminosities, for pure Seyferts, composite Seyferts (both from this paper) and starbursts (from Verma et al. in

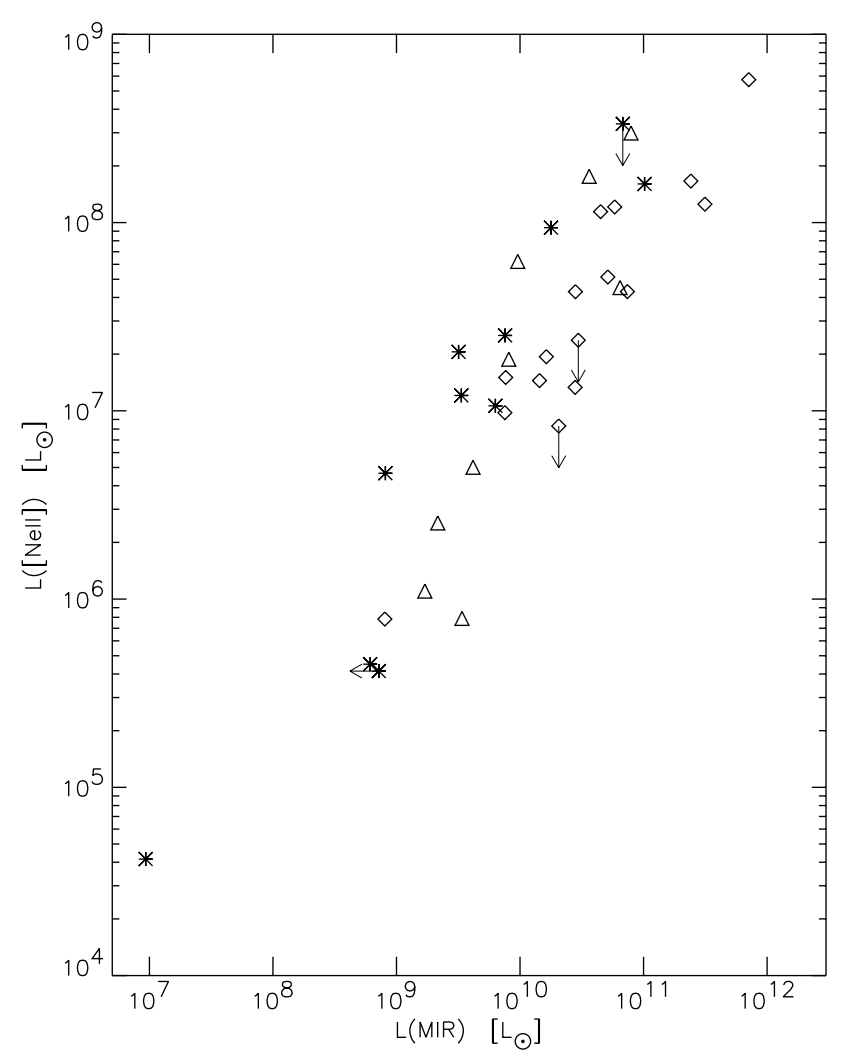

Fig. 13. [Ne II] luminosity vs. MIR luminosity for AGNs (diamonds) and starburst galaxies (stars). Composite sources (see text) are indicated as triangles.

prep.). The object classes are discriminated by plot symbols. For each object class separately, good correlations are observed (see also Prieto et al. 2002 showing a similar analysis for a much smaller Seyfert sample and using ISOPHOT fluxes). The scatter is partly intrinsic but will also include a component due to aperture differences between SWS lines and IRAS continua. This is irrelevant for the AGN NLRs which are smaller than the SWS aperture but may affect some of the more extended starburst emission. The slopes are near 1 suggesting that the spectral properties of each object class do not change much over the overall luminosity range probed by our sample. Comparing different object classes, both Figs. 12 and 14 clearly reflect the much stronger [O IV] emission of Seyferts compared to starbursts (Genzel et al. 1998; Lutz et al. 1998). The composite Seyferts tend to fall between pure Seyferts and starbursts, again confirming our classification based on PAH emission, and consistent with the fine structure line ratios (Sects. 6 and 7). As seen in Fig. 15 and already noted by Genzel et al. (1998), the ratio of [Ne II] and FIR luminosity is very similar in Starbursts and AGNs. The difference in [NeII]/MIR between Seyferts and starbursts is somewhat larger (Fig. 13), due to the moderately MIR-brighter AGN SEDs (e.g., de Grijp et al. 1985, 1987). The small variations in the ratio of [Ne II] and infrared luminosities make this line a useful reference line for comparison of line ratios between different types of galaxies (see below).

A possible test for the origin of the AGN far-infrared emission is to compare the quality of the correlations between 


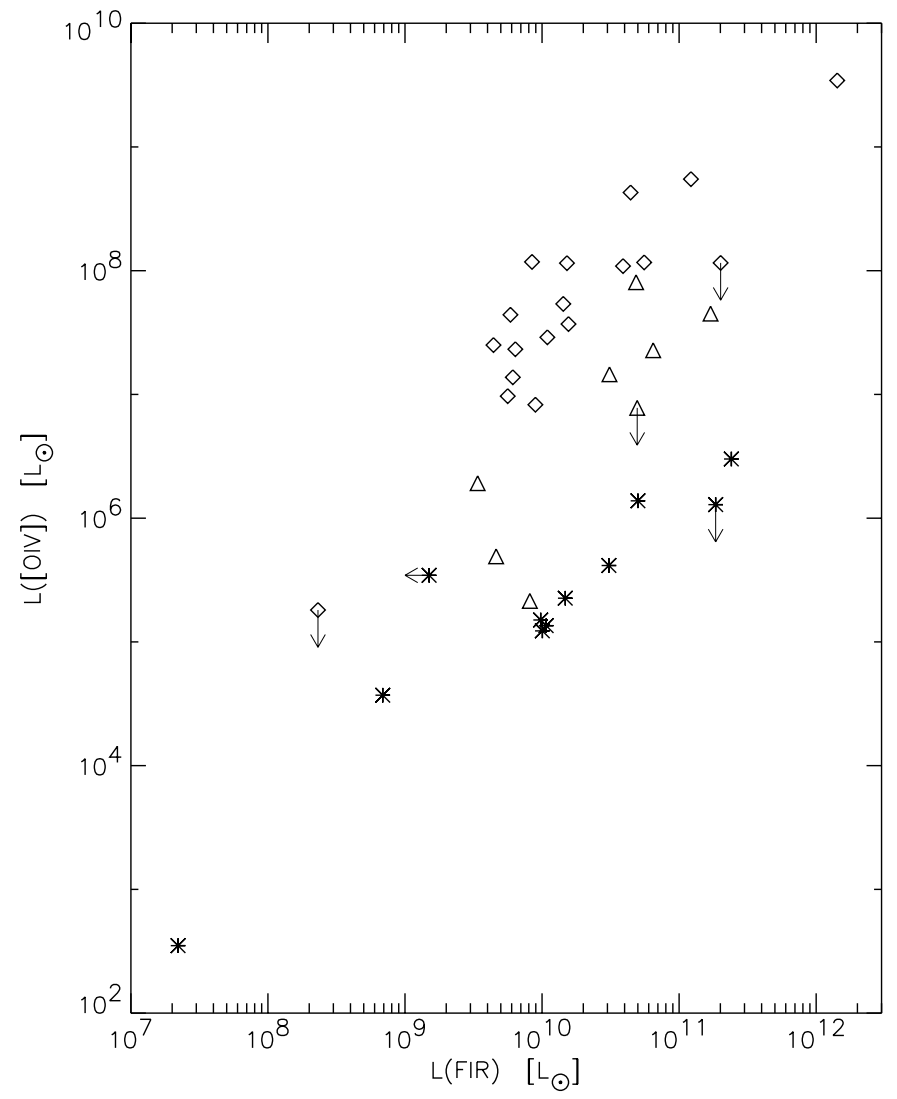

Fig. 14. [O IV] luminosity vs. FIR luminosity for AGNs (diamonds) and starburst galaxies (stars). Composite sources (see text) are indicated as triangles.

[OIV] (assumed to trace AGN luminosity) and MIR/FIR. If the AGN MIR emission were AGN heated but not the FIR emission, one would expect less scatter in the ratio [O IV]/MIR than in $[\mathrm{OIV}] /$ FIR. Figure 16 indeed suggests the correlation with mid-infrared emission to be better, but the effect is not strong (see also the similar conclusion of Prieto et al. 2002). Partly, the small magnitude of the effect may be simply due to the scatter in the "reference" ratio [O IV]/MIR. Even if [O IV] were a perfect measure of AGN power (which it clearly is not), the orientation effects and larger scale contributions to the mid-IR AGN emission (Clavel et al. 2000) would cause some scatter in the left panel of Fig. 16. These results are consistent with a scenario where the mid-infrared luminosity in AGNs is dominated by the active nucleus, whereas the far-infrared luminosity is mainly produced by star formation. The considerable correlation between [O IV] and far-infrared luminosities in Seyferts (Fig. 14) would then simply reflect a starburst-AGN connection in which starburst power and AGN power are linked. There is plenty of evidence reported in the literature for such a connection. For instance there could be a feedback mechanism that controls (circum-)nuclear star formation and Black Hole accretion rate. A more trivial link could simply be the common gas consumption habits of starbursts and AGNs (see e.g. Cid Fernandes et al. 2001). On the other hand, we can not exclude other scenarios in which, for example, the FIR luminosity of AGNs is powered by the active nuclei. The relation of the coronal line fluxes to the

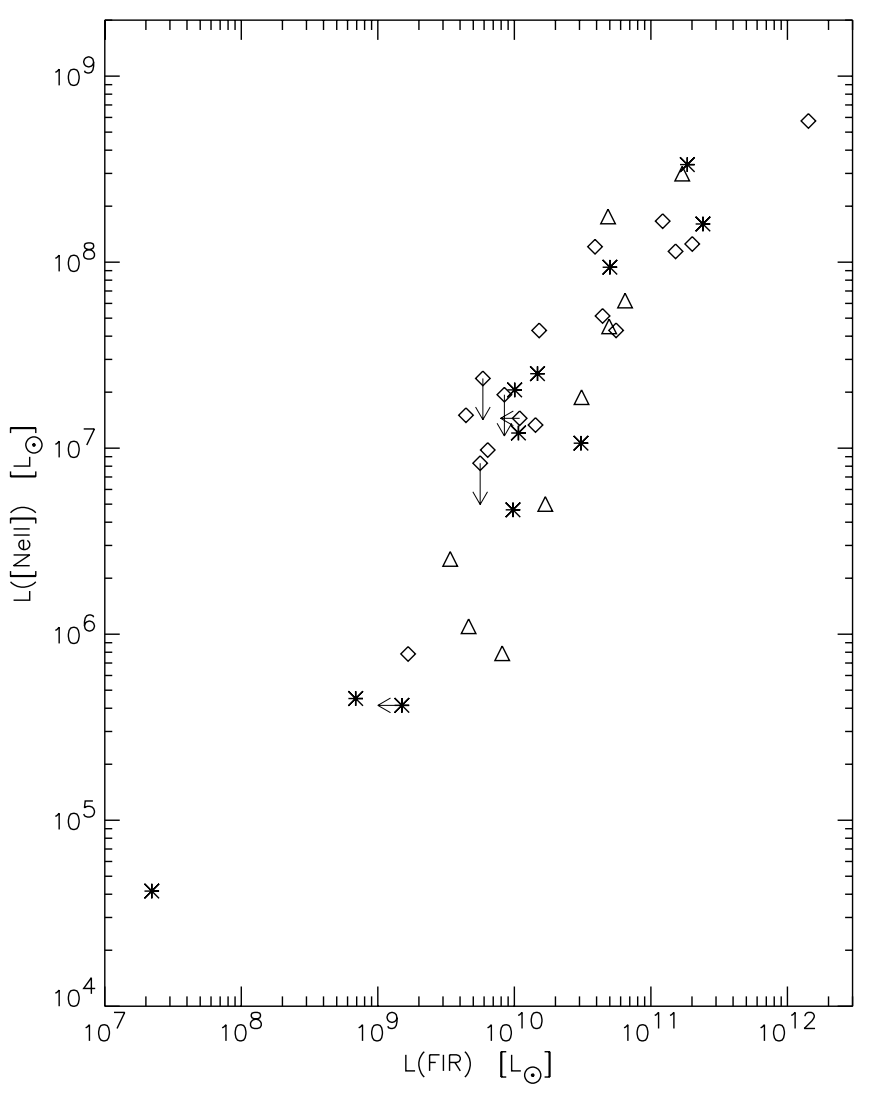

Fig. 15. [Ne II] luminosity vs. FIR luminosity for AGNs (diamonds) and starburst galaxies (stars). Composite sources (see text) are indicated as triangles.
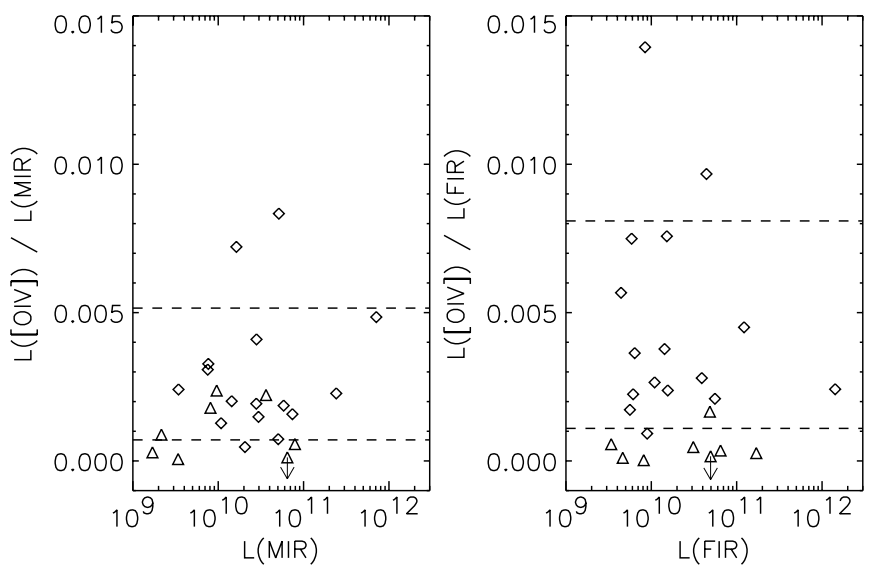

Fig. 16. [O IV] luminosity vs. MIR luminosity (left) and vs. FIR luminosity (right) for pure AGNs (diamonds) and composite sources (triangles). The $\pm 1 \sigma$ range around the average luminosity ratios (computed without composite sources) is marked with dashed lines.

mid/far infrared continuum fluxes does not allow us directly to quantify the AGN contribution to the total luminosity of their host galaxies with sufficient accuracy. However, the constancy of the [Ne II]-to-FIR ratio can be used to construct models for the AGN/starburst contribution to $L_{\mathrm{Bol}}$ from the ratios of MIR lines to [Ne II]. In Figs. 7 and 8 (right hand $y$-axes) we show a simple linear mixing model, using the 


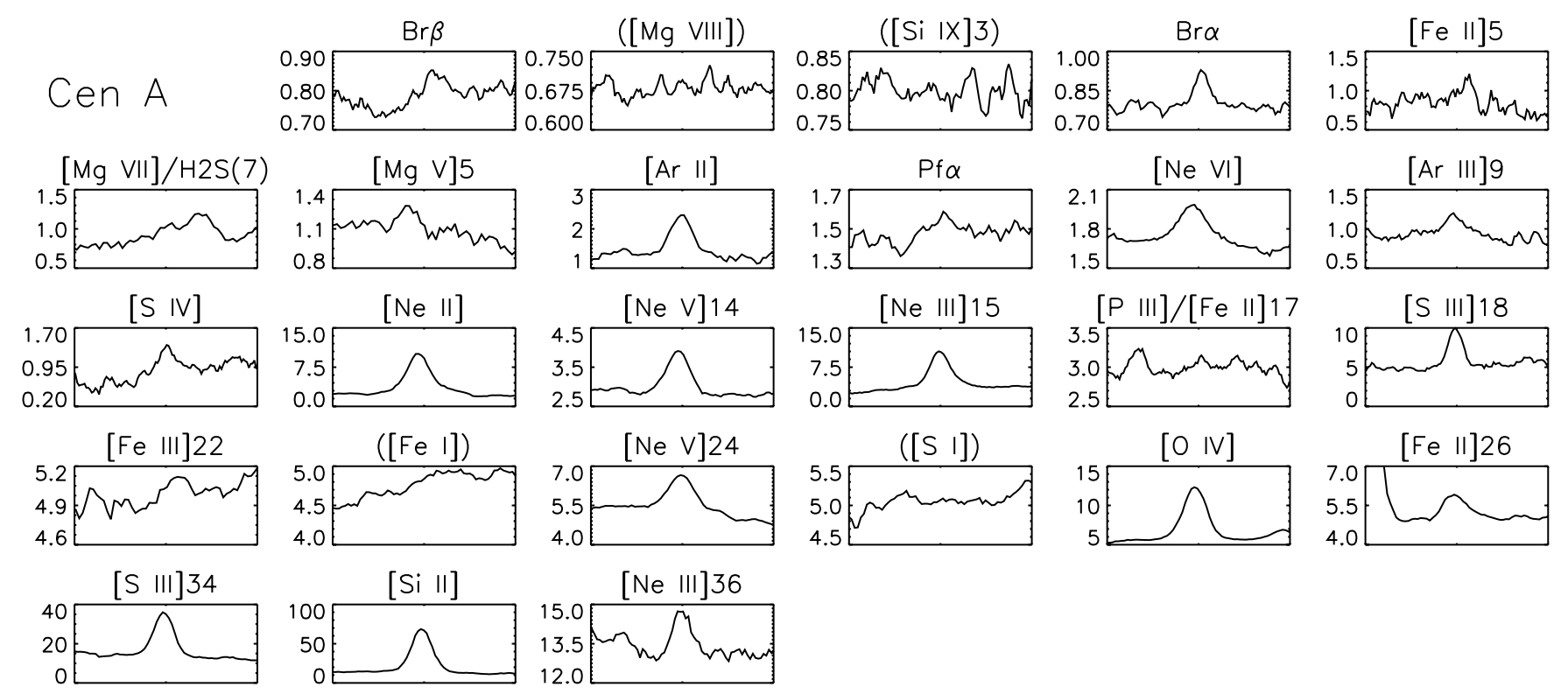

\section{Circinus}
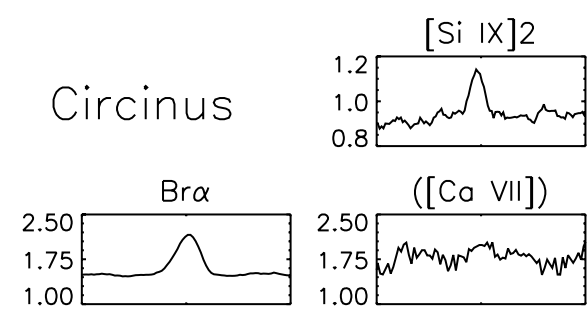

$[\mathrm{Mg} \mathrm{VIII} / \mathrm{H} 2 \mathrm{~S}(7)$
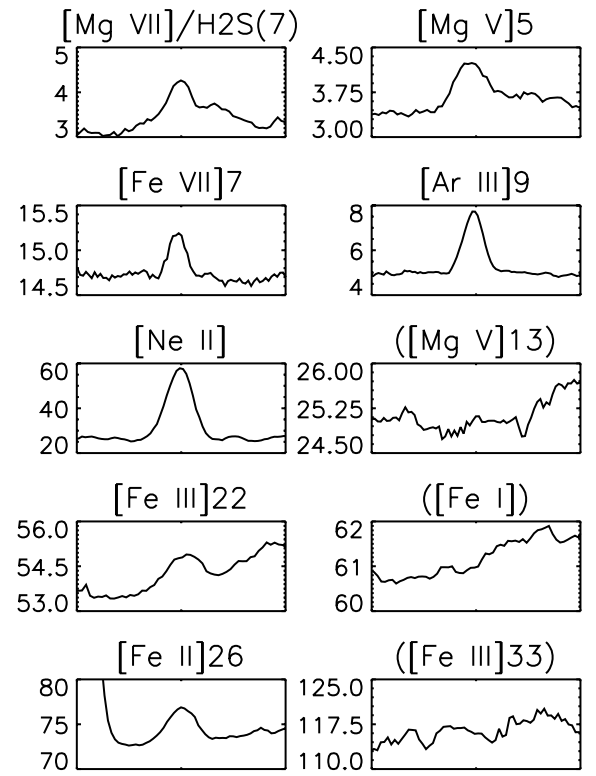
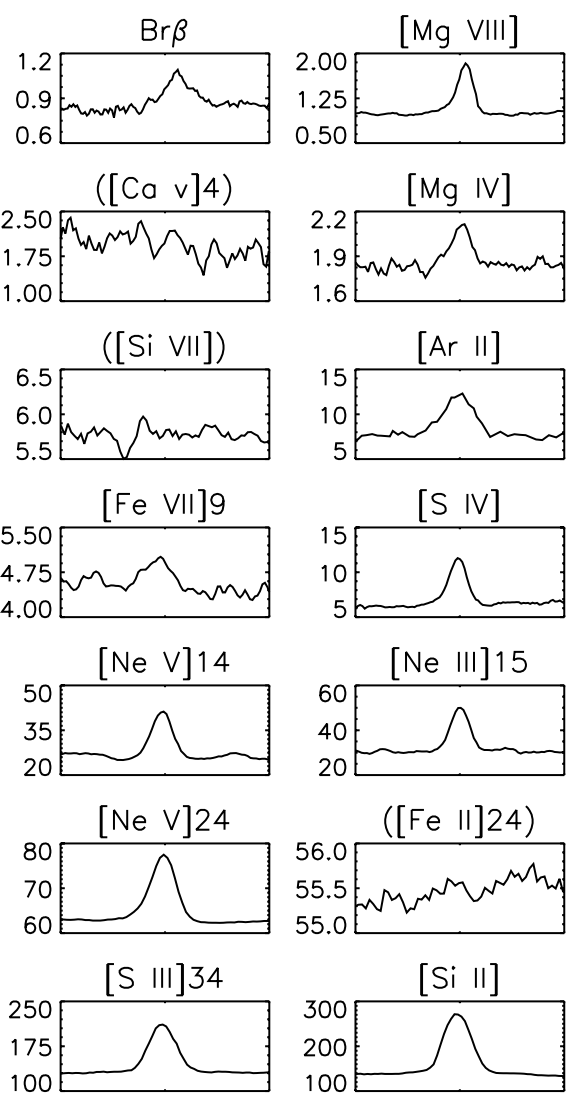
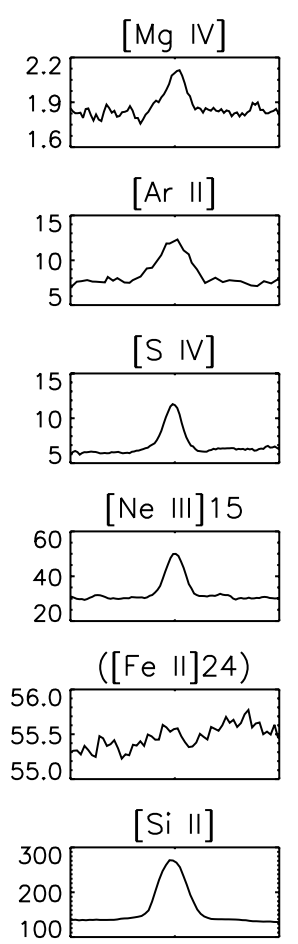
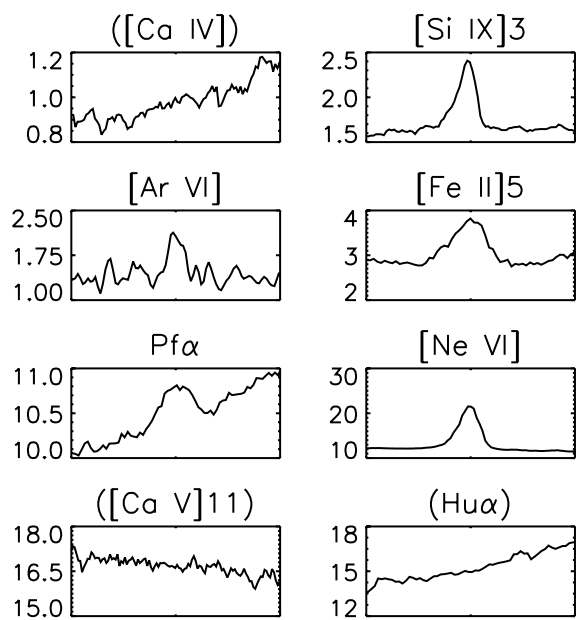

$[\mathrm{Ne} \mathrm{Vl}]$

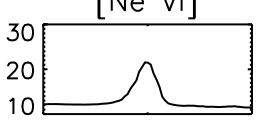

$(\operatorname{Hu} \alpha)$

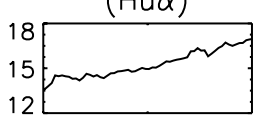

$[\mathrm{P} \| 11] ? /([\mathrm{Fe} \|] 17)$
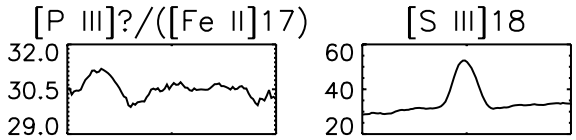

[O IV]
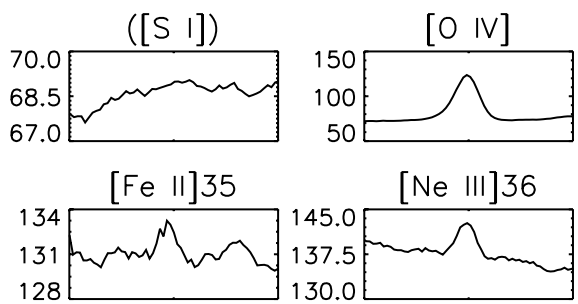

Fig. 17. ISO-SWS spectra of Centaurus A and Circinus. Flux densities in [Jy] for a velocity range of $\pm 1200 \mathrm{~km} \mathrm{~s}^{-1}$ around the respective systemic velocity $\left(v_{\text {sys }}=c z\right.$, see Table 1$)$. Identifications in brackets denote undetected lines with upper flux limits only.

respective median ratios of $[\mathrm{OIV}] /[\mathrm{Ne} \mathrm{II}]$ and $[\mathrm{Ne} \mathrm{V}] /[\mathrm{NeII}]$ for the pure AGNs and the pure starbursts in our sample (see figure caption). Those sources that we have identified as composite sources by means of their PAH spectrum (or their position in Fig. 6) lie in the 10 to 50 percent domain of these mixing curves. When applying such simple models to individual sources one should keep in mind that there is some scatter in the underlying relations, and the derived contributions in percent should not be taken too literally. For statistical studies, however, these models provide a valuable means of quantifying the AGN contribution to the luminosity of dusty, composite objects. 


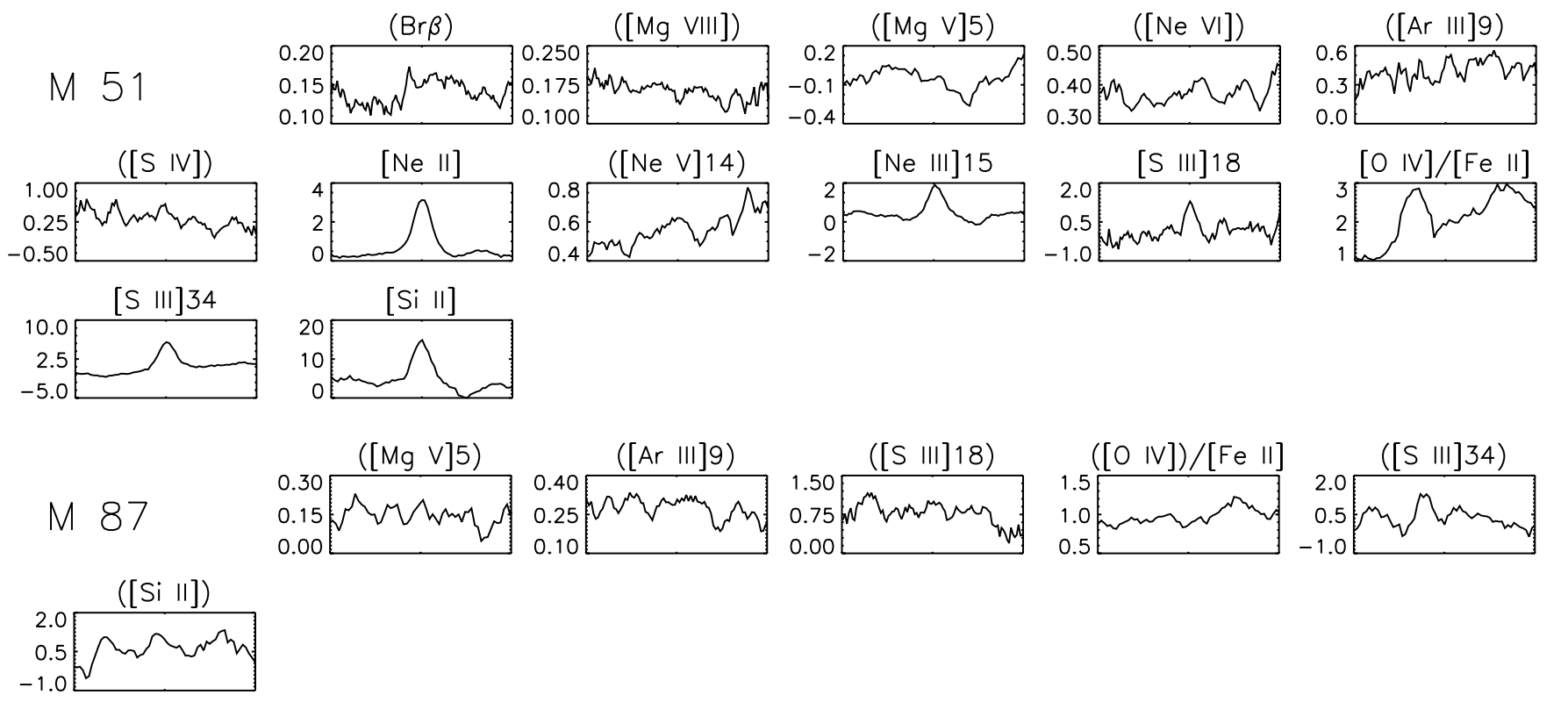

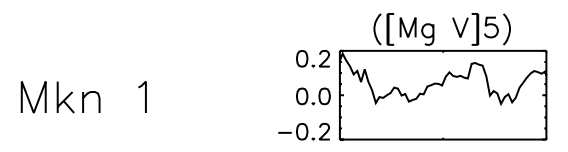
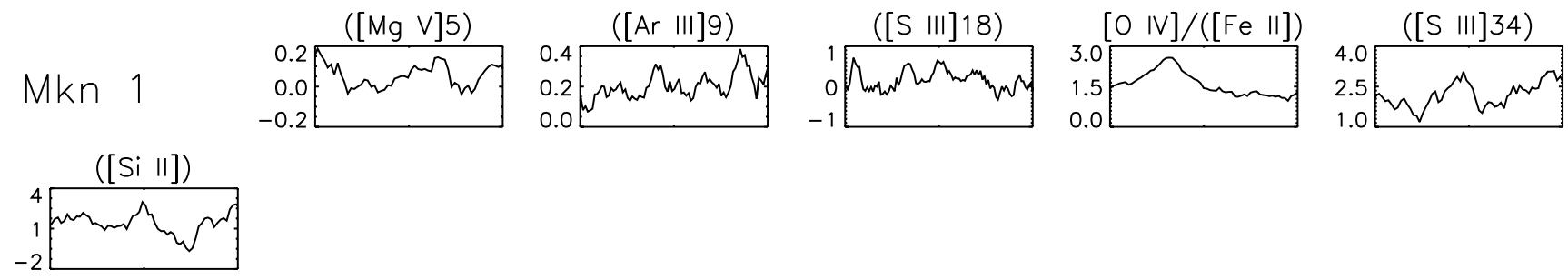

Mkn3

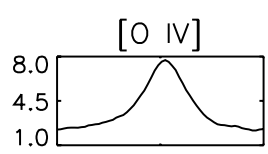

$M k n 6$
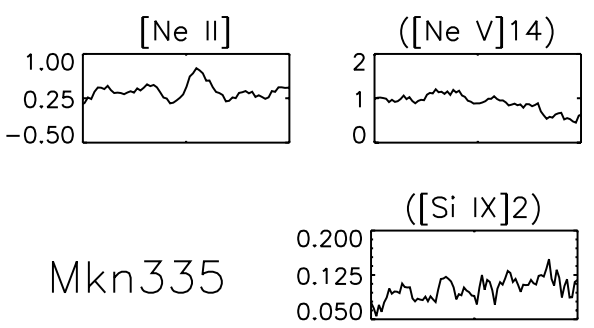

$([\mathrm{Ne} V I])$
0.30
0.20
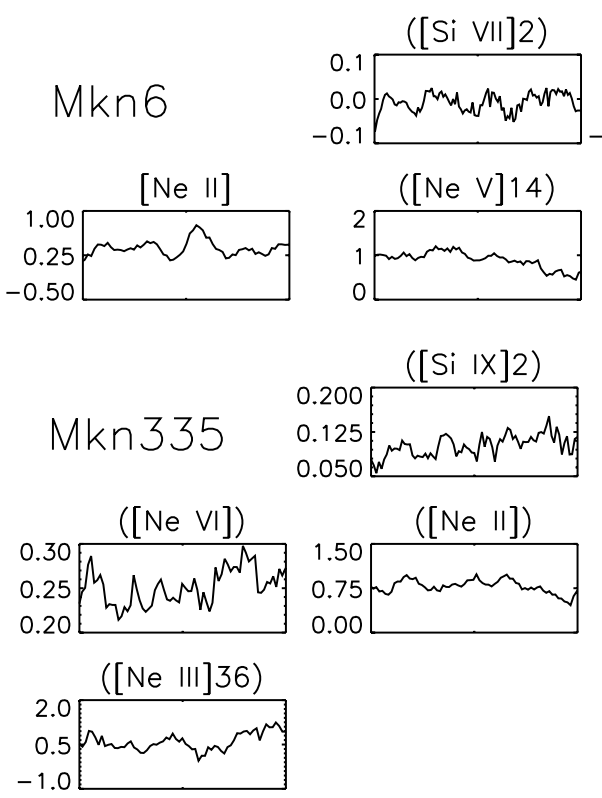
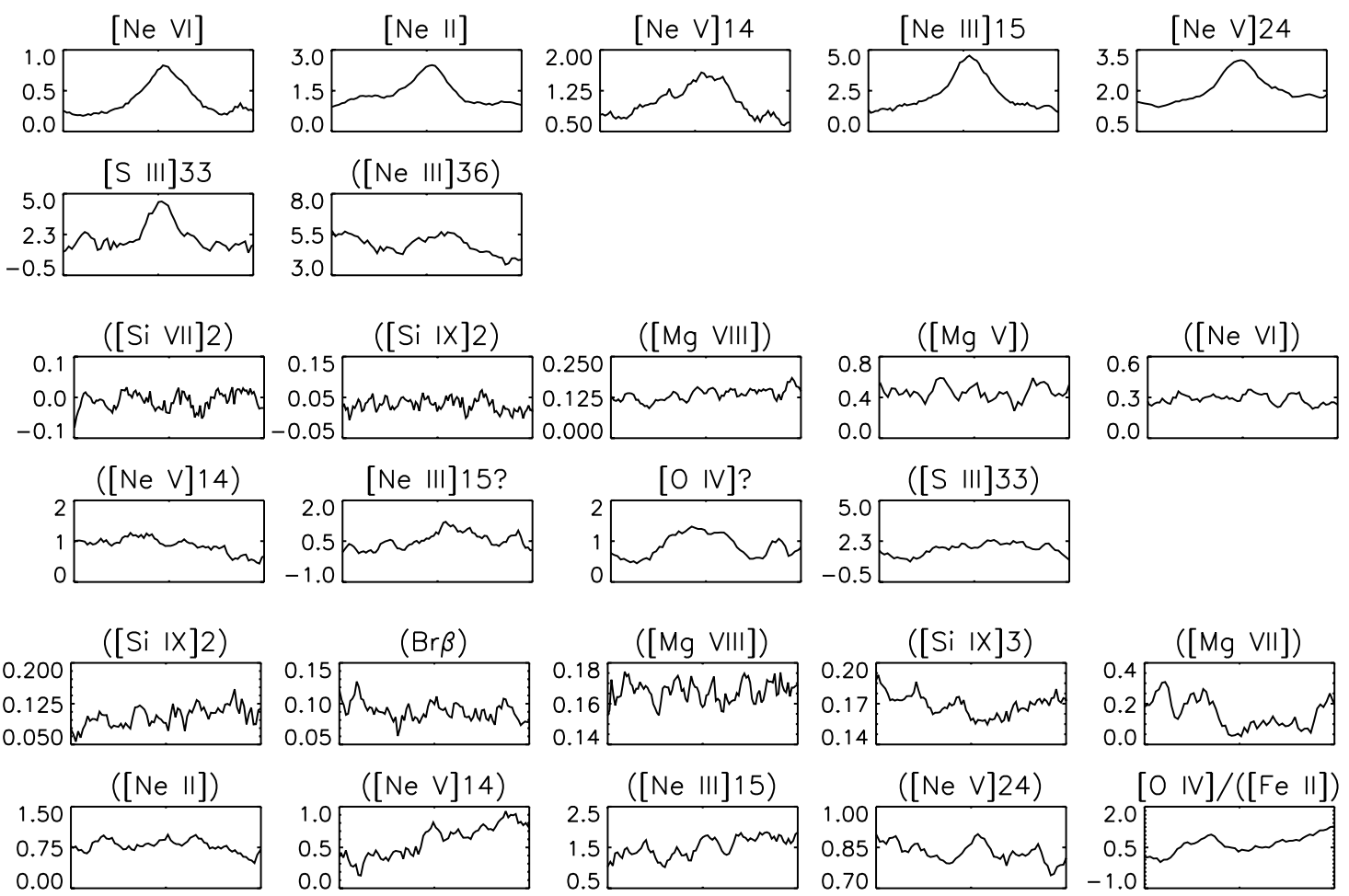

$[\mathrm{O} \mathrm{IV}] /([\mathrm{Fe} \|])$

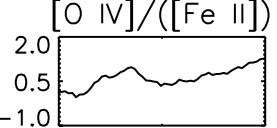

Fig. 18. Further SWS spectra. Same units as in Fig. 17. The range for the combined [O IV]/[Fe II] spectra is $[-700,+1700] \mathrm{km} \mathrm{s}^{-1}$ around $[\mathrm{O} I V]$. 


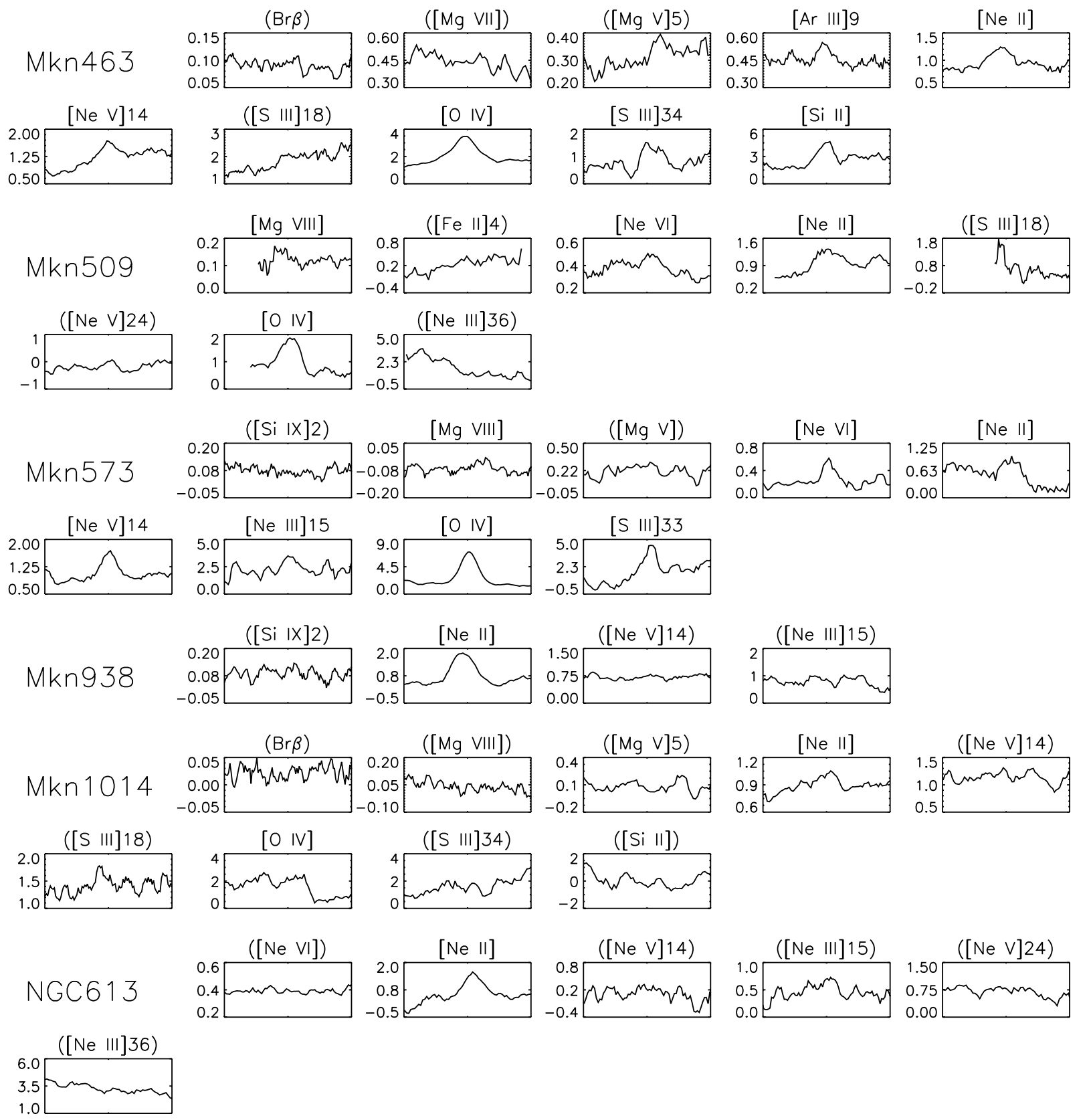

Fig. 19. Further SWS spectra. Same units as in Fig. 17.

\section{Summary}

We have presented an inventory of mid-infrared (MIR) lines detected in medium resolution $(R \sim 1500)$ ISO-SWS $2.4-45 \mu \mathrm{m}$ spectra of a sample of 29 galaxies with active nuclei. This data set is rich in fine structure emission lines tracing the narrow line regions and (circum-)nuclear star formation regions, and it provides a coherent spectroscopic reference for future extragalactic studies in the mid-infrared. From the [Ne V] 14/24 $\mu \mathrm{m}$ line ratios we conclude that all targets in our sample with measurable [Ne V] emission have relatively low NLR electron densities (a few 100 to a few $1000 \mathrm{~cm}^{-3}$ ). Electron temperatures are difficult to derive. There are indications for high (20000$30000 \mathrm{~K}$ ) electron temperatures in the part of the narrow line

region sampled by $E_{\text {ion }} \sim 100 \mathrm{eV}$ species, but there is considerable uncertainty on extinction corrections and atomic data which prevents firm conclusions.

A comparison of the observations to photoionization models showed that all objects are consistent with standard photoionization models, i.e. the spectral energy distribution of their central ionizing source can be explained by a normal power law. These simple comparisons can not, however, distinguish between simple power laws and more complex models of a "Big UV Bump" and possibly absorption. The detailed modeling required for such an analysis is outside the scope of this paper. Within the statistical limitations of our sample we do not 


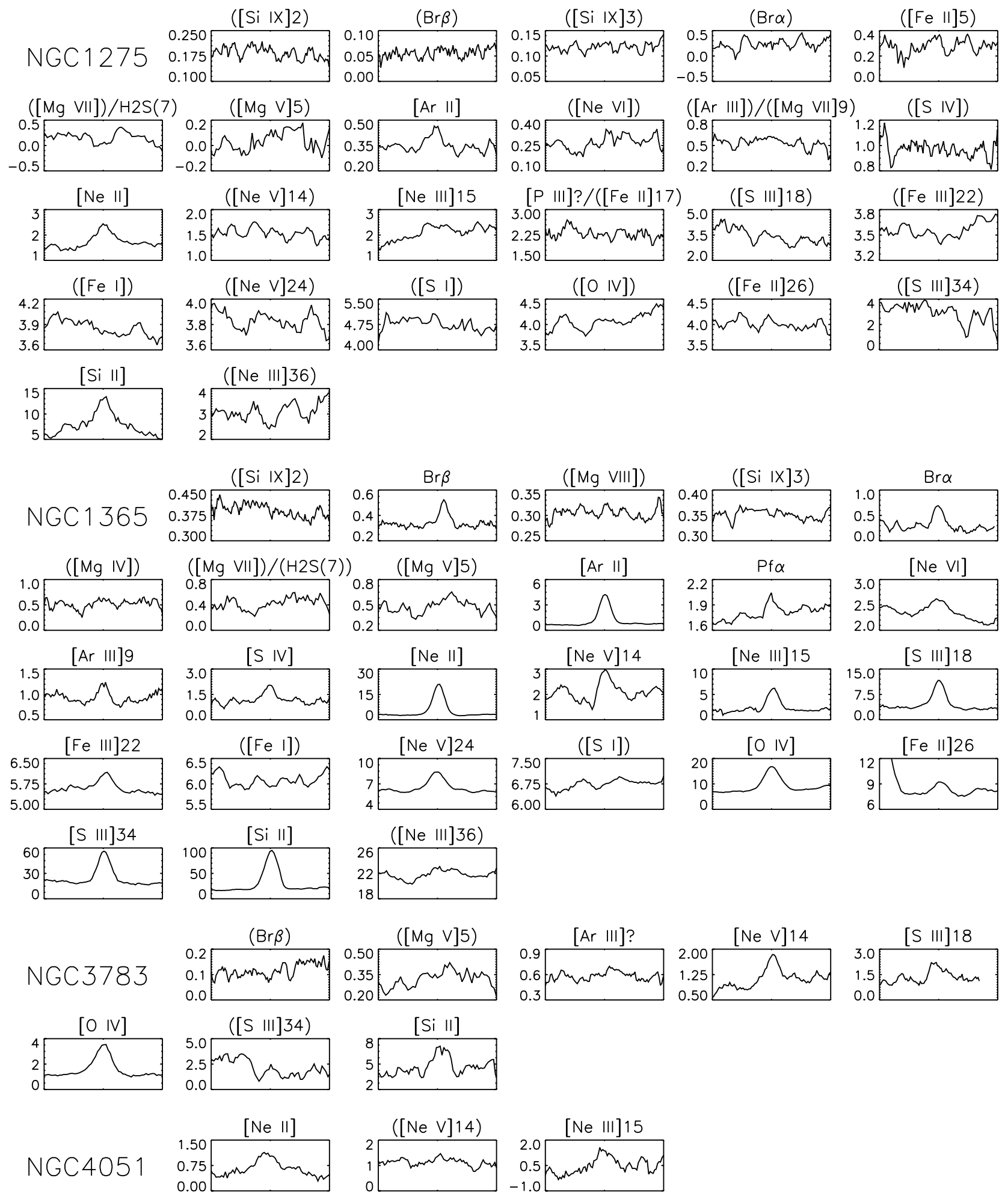

Fig. 20. Further SWS spectra. Same units as in Fig. 17.

see any significant differences of NLR densities or ionization between the different AGN types.

The MIR lines in our data set show various degrees of profile asymmetries and different levels of similarities to their optical counterparts. It appears that the cause for the line profile asymmetries in Seyfert galaxies is a complex mixture of extinction by dust on different spatial scales, sub-structure and true asymmetries in the spatial distribution of NLR clouds.

We have used the line ratios of $[\mathrm{NeVI}] /[\mathrm{OIV}]$ and $[\mathrm{Ne} \mathrm{VI}] /[\mathrm{NeII}]$ as well as the strength of the PAH features as independent indicators for the identification of composite sources, i.e. sources in which star-forming regions 

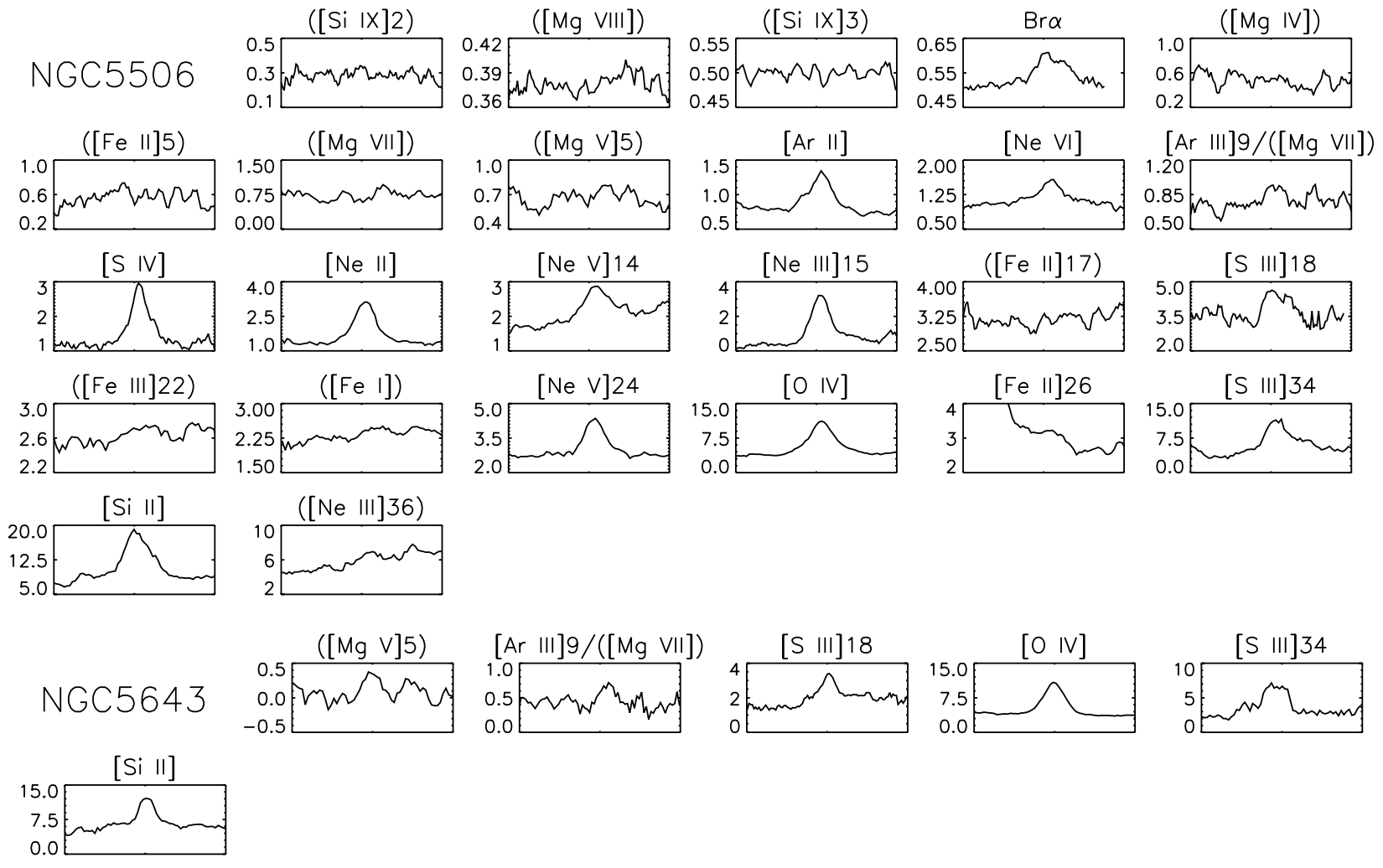

NGC7469
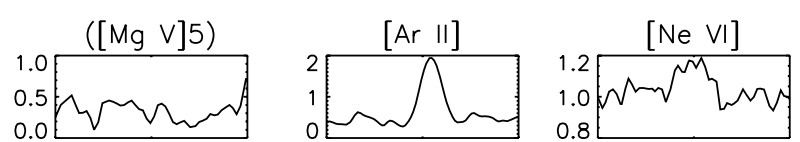

$[\mathrm{Ar} I I I] 9 ? /(\mathrm{Mg} \mathrm{VII})$

$[\mathrm{S} I \mathrm{~V}]$

$[\mathrm{Ne} I 1]$
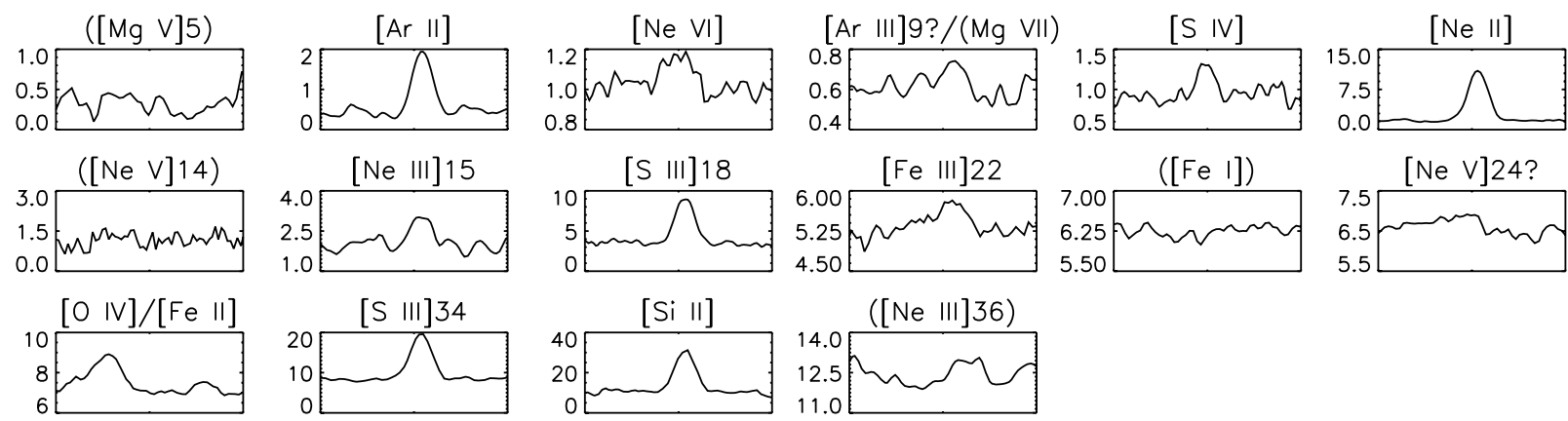

Fig. 21. Further SWS spectra. Same units as in Fig. 17. The range for the combined $[\mathrm{OIV}] /[\mathrm{Fe}$ II $]$ spectrum is $[-700,+1700] \mathrm{km} \mathrm{s}^{-1}$ around [O IV].

within the aperture contribute to the fluxes of low lying fine structure lines. As a new tool for determining the properties of dusty galaxies we provide mid-IR line ratio diagrams, like [O IV] $25.9 \mu \mathrm{m} /[\mathrm{Ne} \mathrm{II}] 12.8 \mu \mathrm{m}$, which can be used to distinguish between emission excited by active nuclei and emission from (circum-nuclear) star forming regions. In addition, we present two-dimensional diagnostic diagrams that are fully analogous to classical optical diagnostic diagrams, but better suited for objects with high extinction. Several combinations of lines like [O IV], [S IV], [Si II], or [Fe II], normalized to hydrogen recombination lines, can be used, allowing to adapt to redshift range or wavelength coverage of different detectors.

The high ionization lines like [O IV], [Ne V], or [Ne VI] in Seyfert galaxies are well correlated with both the mid-IR and far-IR continuum radiation, the latter correlation showing a somewhat larger scatter than the former. Low ionization lines, which might be significantly affected by star formation, show similar correlations. In particular the [Ne II]-to-FIR correlation is very similar in both Seyfert and starburst galaxies. On the one hand, this makes it difficult to quantify the dominant contributor to the bolometric luminosities of Seyfert host galaxies directly from such ratios of MIR lines to FIR continuum. On the other hand, it makes [Ne II] a valuable reference line for comparisons of line ratios between different sources. We have used this to construct simple linear mixing models of AGN contribution to the bolometric luminosities of composite sources for the ratios $[\mathrm{OIV}] /[\mathrm{Ne} \mathrm{II}]$ and $[\mathrm{Ne} \mathrm{V}] /[\mathrm{Ne} \mathrm{II}]$. 




Fig. 22. Further SWS spectra. Same units as in Fig. 17. 

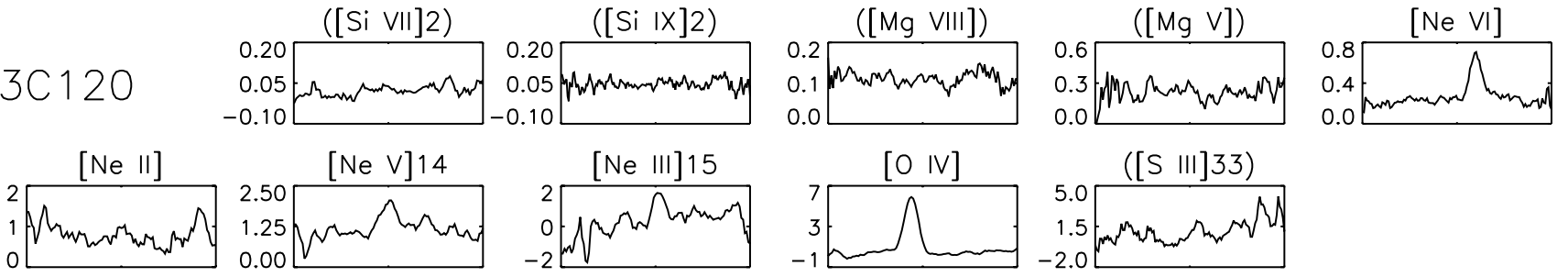

Fig. 23. Further SWS spectra. Same units as in Fig. 17.

Acknowledgements. We wish to thank Julia Morfill for her help with the data reduction. SWS and the ISO Spectometer Data Center at MPE are supported by DLR under grant 50 QI 0202 and by Verbundforschung under grant 50 OR 99137 . This research was supported in part by the German-Israeli Foundation (grant I-0551186.07/97). The ISO Spectral Analysis Package (ISAP) is a joint development by the LWS and SWS Instrument Teams and Data Centers. Contributing institutes are CESR, IAS, IPAC, MPE, RAL and SRON. This research has made use of the NASA/IPAC Extragalactic Database (NED) which is operated by the Jet Propulsion Laboratory, California Institute of Technology, under contract with the National Aeronautics and Space Administration.

\section{References}

Alexander, D. M., Efstathiou, A., Hough, J. H., et al. 1999a, MNRAS, 310,78

Alexander, D. M., Hough, J. H., Young, S., et al. 1999b, MNRAS, 303, L17

Alexander, T., Sturm, E., Lutz, D., et al. 1999, ApJ, 512, 204

Alexander, T., Lutz, D., Sturm, E., et al. 2000, ApJ, 536, 710

Axon, D., Marconi, A., Capetti, A., et al. 1998, ApJ, 496, L75

Baldwin, J. A., Phillips, M. M., \& Terlevich, R. 1981, PASP, 93, 5

Berrington, K. A., Nakazaki, S., \& Norrington, P. H. 2000, A\&AS, 142,313

Binette, L., Wilson, A. S., \& Storchi-Bergman, T. 1996, A\&A, 312, 365

Binette, L., Wilson, A. S., Raga, A., \& Storchi-Bergmann, T. 1997, A\&A, 327, 909

Cameron, M., Storey, J. W. V., Rotaciuc, V., et al. 1993, ApJ, 419, 136

Chiar, J. E., Tielens, A. G. G. M., Whittet, D. C. B., et al. 2000, ISO beyond the peaks: The 2nd ISO workshop on analytical spectroscopy, ed. A. Salama, M. F. Kessler, K. Leech, \& B. Schulz. ESA-SP 456, 45

Cid Fernandes, R., Heckman, T., Schmitt, H., Delgado, R. M. G., \& Storchi-Bergmann, T. 2001, ApJ, 558, 81

Clavel, J., Schulz, B., Altieri, B., et al. 2000, A\&A, 357, 839

Contini, M., \& Viegas, S. M. 2001, ApJS, 137, 75

De Graauw, T., Haser, L. N., Beintema, D. A., et al. 1996, A\&A, 315, L49

de Grijp, M. H. K., Miley, G. K., Lub, J., \& de Jong, T. 1985, Nature, 314,240

de Grijp, M. H. K., Lub, J., \& Miley, G. K. 1987, A\&AS, 70, 95

Desert, F.-X., Boulanger, F., \& Puget, J. L. 1990, A\&A, 237, 215

Genzel, R., Lutz, D., Sturm, E., et al. 1998, ApJ, 498, 579

Genzel, R., \& Cesarsky, C. J. 2000, ARA\&A, 38, 761

Giveon, U., Sternberg, A., Lutz, D., Feuchtgruber, H., \& Pauldrach, A. W. A. 2002, ApJ, 566, 880

González Delgado, R. M., Heckman, T., \& Leitherer, C. 2001, ApJ, 546, 845

Heckman, T. M., Gonzalez-Delgado, R., Leitherer, C., et al. 1997, ApJ, 482, 114
Kessler, M. F., Steinz, J. A., Anderegg, M. E., et al. 1996, A\&A, 315, L27

Kraemer, S. B., Turner, T. J., Crenshaw, D. M., \& George, I. M. 1999, ApJ, 519, 69

Kraemer, S. B., Crenshaw, D. M., \& Gabel, J. R. 2001, ApJ, 557, 30

Kriss, G. A., Davidsen, A. F., Blair, W. P., et al. 1992, ApJ, 392, 485

Lahuis, F., Wieprecht, E., Bauer, O. H., et al. 1998, Astronomical Data Analysis Software and Systems VII, 7, ASP Conf. Ser. 145, 224

Lennon, D. J., \& Burke, V. M. 1994, A\&AS, 103, 273

Lutz, D., Kunze, D., Spoon, H. W. W., \& Thornley, M. D. 1998, A\&A, 333, L75

Lutz, D., Genzel, R., Sturm, E., et al. 2000, ApJ, 530, 733

Lutz, D., Sturm, E., Genzel, R., et al. 2000, ApJ, 536, 697

Marconi, A., van der Werf, P. P., Moorwood, A. F. M., \& Oliva E. 1996, A\&A, 315, 335

Moorwood, A. F. M., Lutz, D., Oliva, E., et al. 1996, A\&A, 315, L109

Nussbaumer, H., \& Rusca, C. 1979, A\&A, 72, 129

Oliva, E., Salvati, M., Moorwood, A. F. M., \& Marconi, A. 1994, A\&A, 288, 457

Oliva, E., Moorwood, A. F. M., Drapatz, S., Lutz, D., \& Sturm, E. 1999, A\&A, 343, 943

Prieto, M. A., \& Viegas, S. M. 2000, ApJ, 532, 238

Prieto, M. A., Perez Garcia, A. M., \& Rodriguez Espinosa, J. M. 2002 [astro-ph/0109083]

Rigopoulou, D., Spoon, H. W. W., Genzel, R., et al. 1999, AJ, 118, 2625

Rigopoulou, D., Kunze, D., Lutz, D., Genzel, R., \& Moorwood, A. F. M. 2002, A\&A, 389, 374

Salas, J. B., Pottasch, S. R., Beintema, D. A., \& Wesselius, P. R. 2001, A\&A, 367, 949

Sanders, D. B., \& Mirabel, I. F. 1996, ARA\&A, 34, 749

Siebenmorgen, R., Moorwood, A., Freudling, W., \& Kaeufl, H. U. 1997, A\&A, 325, 450

Spinoglio, L., \& Malkan, M. A. 1992, ApJ, 399, 504

Spinoglio, L., Benedettini, M., De Troia, G., et al. 2000, ISO beyond the peaks: The 2nd ISO workshop on analytical spectroscopy, ed. A. Salama, M. F. Kessler, K. Leech, \& B. Schulz, ESA-SP 456, 261

Spoon, H. W. W., Keane, J. V., Tielens, A. G. G. M., et al. 2002, A\&A, 385,1022

Sturm, E., Lutz, D., Genzel, R., et al. 1996, A\&A, 315, L133

Sturm, E., Bauer, O. H., Brauher, J., et al. 1998, Astronomical Data Analysis Software and Systems VII, 7, ASP Conf. Ser. 145, 161

Sturm, E., Alexander, T., Lutz, D., et al. 1999, ApJ, 512, 197

Tran, Q. D., Lutz, D., Genzel, R., et al. 2001, ApJ, 552, 527

Veilleux, S., \& Osterbrock, D. E. 1987, ApJS, 63, 295

Veilleux, S. 1991, ApJS, 75, 357

Veilleux, S., Kim, D.-C., Sanders, D. B., Mazzarella, J. M., \& Soifer, B. T. 1995, ApJS, 98, 171

Veilleux, S., Kim, D.-C., \& Sanders, D. B. 1999, ApJ, 522, 113

Voit, G. M. 1992, ApJ, 399, 495

Vrtilek, J. M., \& Carleton, N. P. 1985, ApJ, 294, 106.

Whittle, M. 1985, MNRAS, 213, 1

Wieprecht, E., Lahuis, F., Bauer, O. H., et al. 1998, Astronomical Data Analysis Software and Systems VII, 7, ASP Conf. Ser. 145, 279 